\title{
SEX DIFFERENCES IN SENSORIMOTOR MU \\ RHYTHMS DURING SELECTIVE ATTENTIONAL \\ PROCESSING
}

By

Christina M. Popovich

A thesis submitted in conformity with the requirements

for the degree of Master of Science

Graduate Department of Institute of Medical Sciences, Department of Medicine University of Toronto

(C) by Christina M. Popovich, 2010 


\section{Sex Differences in Sensorimotor Mu Rhythms During Selective Attentional Processing}

Master's of Science, 2010

Christina Popovich

Institute of Medical Science

University of Toronto

\section{ABSTRACT}

Magnetoencephalography was used to investigate the effect of directed attention on changes in sensorimotor mu (8-12Hz) response (mu reactivity) to non-painful electrical stimulation of the median nerve in healthy adults. Results indicated attentionrelated sex differences in mu reactivity, with females showing i) prolonged mu suppression when attending to somatosensory stimuli indicating active processing of the sensory stimuli; ii) task-dependent attentional modulation of the mu response, which was absent in males, and iii) a trend for greater neuronal excitability of the primary somatosensory region suggesting greater physiological responsiveness to stimulation overall. Sex-related differences in attentional modulation of sensorimotor rhythms suggest that females and males use different top-down control strategies when processing somatosensory information. These sex differences in attention may underlie welldocumented sex-related biases in pain processing wherein females typically report greater sensitivity to experimental and clinical pain. 


\section{ACKNOWLEDGEMENTS}

I would like to thank and extend my sincere gratitude and appreciation to my supervisor, Dr. Rosemary Tannock. She has been exceptionally supportive and her guidance, knowledge, and mentorship made this project not only possible, but also propelled it into new and exciting directions. I would like to thank members of my committee, Dr. Douglas Cheyne, Dr. Randy McIntosh, and Dr. Mary Pat McAndrews for their advice, expertise, and support of this project. Thank you for your co-operation, encouraging words of wisdom and for donating your time to this thesis research. A special thank you to Dr. Colleen Dockstader, who actively helped mentor me throughout the duration of this project and who was always exceptionally supportive and willing to share her knowledge and technical expertise with me. Finally, I would like to extend a sincerely deep gratitude to my parents, Anne and Dan, and to my sisters, Suzanne and Denise, for all of their support in pursuing my education and believing I will always succeed. Thank you for always being there to share the great and the frustration moments. 


\section{TABLE OF CONTENTS}

ABSTRACT

ACKNOWLEDGEMENTS iii

LIST OF FIGURES $\quad$ v

LIST OF ABBREVIATIONS

PART I: LITERATURE REVIEW

SECTION I: WHAT IS THE FUNCTIONAL SIGNIFICANCE OF CORTICAL

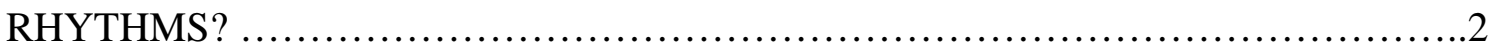

1.1.1. Definition and measurement of cortical rhythms.....................

1.1.2. Attentional modulation of cortical rhythms..........................6

1.1.3. Sex differences in selective attention....................................9

SECTION II: THE SOMATOSENSORY CORTEX................................12

1.2.1. Sensorimotor Mu Rhythms.....................................13

1.2.2. Mu Rhythms and the empathetic response hypothesis..................17

1.2.3. Sex differences in mu modulation....................................19

1.2.4. Advantages of using MEG and MNS to study somatosensory processing..................................................22

SECTION III: THE RATIONALE FOR THE PRESENT STUDY ......................24

1.3.1 Aims and hypotheses................................................26

PART II: SEX DIFFERENCES IN SENSORIMOTOR MU RHYTHMS DURING

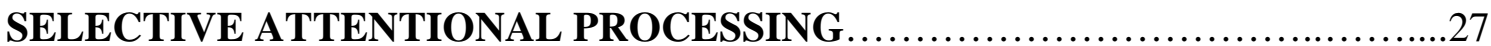

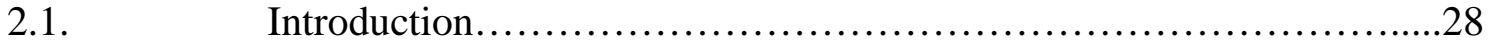

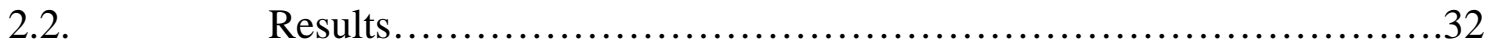

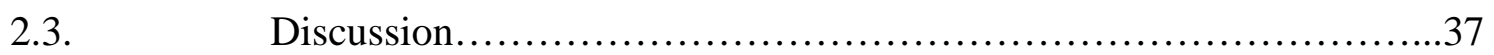

2.4. Materials and Methods...........................................42

PART III: GENERAL DISCUSSION ......................................... 49

3.1. General Discussion Overview..................................50

3.2. The sex-specific neurocognitive theory of attention..................50

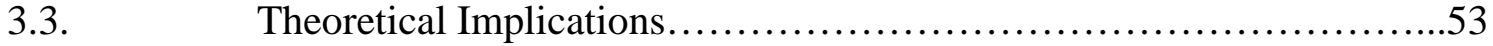

3.4. Clinical Implications.............................................

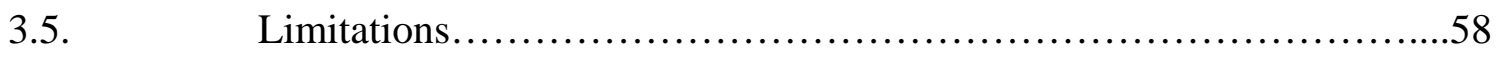

3.6. $\quad$ Future Directions..............................................60 


\section{LIST OF FIGURES}

\section{PART II: SEX DIFFERENCES IN SENSORIMOTOR MU RHYTHMS DURING SELECTIVE ATTENTIONAL PROCESSING}

Figure 1A. Female vs. Male SI Localization: Attend to tactile stimuli...............................80

Figure 1B. Female vs. Male SI Localization: Attend away from tactile stimuli. ..............81

Figure 2. An effective paradigm for observing SI attentional effects. ..............................82

Figure 3. Females show prolonged somatosensory activation when attending to

MNS

Figure 4. Grand averaged sex-specific mu values following the initial three stimuli of a

train. .84

Figure 5. Sex-specific SI modulation effects of self-directed attention to the 4s MNS break interval...

Figure 6. Grand-averaged sex-specific mu reactivity values from directed-attention to the 4 s MNS break interval. . .86 


\section{LIST OF ABBREVIATIONS}

(ADHD) Attention Deficit Hyperactivity Disorder

(ANOVA) analysis of variance

(ANT) Attention Network Test

(BOLD) blood oxygenated level dependence

(EEG) Electroencephalography

(ERD) event-related desynchrony

(ERPs) event-related potentials

(ERS) event-related synchrony

(fMRI) functional magnetic resonance imaging

(MEG) Magnetoencephalography

(MNS) median nerve stimulation

(MRI) magnetic resonance imaging

(MRT) mental rotation tasks

(RT) reaction time

(PDM) primary dysmenorrheal

(SAM) synthetic aperture magnetometry

(SI/SII) primary/secondary somatosensory cortices

(SPSS) statistical package for the social sciences

(TFRs) time frequency response plots

(VEP) visual evoked potential 


\section{PART I \\ LITERATURE REVIEW}




\section{SECTION I: DEFINITION AND MEASUREMENT OF CORTICAL OSCILLATIONS?}

Cognitive behavioural functions are performed by both humans and animals on a daily basis. These functions may include (but are not limited to): attentional processing, short term and/or long term memory processes, as well as performance of basic and/or complex cognitive and motor actions. Successful completion of these and other tasks requires temporally cohesive communication between neural populations in order for the incoming sensory codes to be processed efficiently (Neuper\& Pfurtscheller, 2001). Functional neuroimaging techniques such as electroencephalography (EEG) and magnetoencephalography (MEG) have enabled cognitive neuroscientists to examine the spatiotemporal dynamics of neurophysiological factors, including rhythmic patterns of cortical neural activity. These technological advances in neuroscience methodology have demonstrated that the temporal relationship between neurons can occur either in synchrony (i.e. the firing rate of two or more neurons is correlated such that the firing of one neuron increases the probability that other neurons will fire in a similar manner), or in desychrony (i.e. neural populations fire randomly and independently of one another). When large neural assemblies respond to incoming sensory codes in one of these ways, cortical rhythms are produced.

Neural networks can display different states of synchrony, with rhythmic activity occurring at different frequencies (Lopes da Silva, 1991). Moreover, the frequency rate of neural communication is negatively correlated with the amplitude of the oscillations 
(Lopes da Silva \& Pfurtscheller, 1999), and the amplitude of the oscillation is proportional to the number of synchronously active neural assemblies (Elul, 1972). Several cortical rhythms have characteristic frequency ranges and spatial distributions which modulate with different behavioural states. For example, large neuronal groups typically produce high amplitude, low frequency rhythms (delta (0-4 Hz), theta (4-8 Hz)), whereas faster rhythms (alpha (8-12 Hz), beta $(13-30 \mathrm{~Hz})$, gamma $(30+\mathrm{Hz})$ exhibit low amplitude, high frequency characteristics and occur when locally restricted neurons fire in synchrony with each other (Lopes de Silva \& Pfurtscheller, 1999). The general belief in neurophysiological research is that cortical rhythms are associated with a wide-range of cognitive behavioural functions. Thus, decades of research has been devoted to gaining a better understanding of neural communication, its involvement in producing spatiotemporal cortical rhythm dynamics, and its role in understanding how the brain interprets, processes, and responds to sensory stimuli in our environment.

\subsubsection{Definition and measurement of cortical rhythms}

The general assumption of cortical rhythms is that populations of neurons exist in varying states of synchrony as they respond to externally or internally generated events. Synchrony is defined as the temporal relation between populations of neurons (Salinas \& Sejnowski, 2001). Event-related desynchrony (ERD) and event-related synchrony (ERS) phenomena are thought to represent decreases and increases, respectively, in synchronization within a specific frequency range in relation to an event (Neuper \& Pfurtscheller, 2001). ERD expressed in certain frequency bandwidths (i.e. alpha/ mu), is thought to reflect cortical activation and vigilant processing of the task-relevant sensory 
information, whereas ERS in this bandwidth may represent a deactivated/inhibited cortical area or disengagement from sensory processing (Lopes da Silva \& Pfurtscheller, 1999; Neuper \& Pfurtscheller, 2001). These neural dynamics are believed to be particularly relevant for understanding various cognitive behaviours and functions including attentional processing - the focus of the following research project.

Cortical rhythms are delineated based on the frequency rate (Hz/sec). Slow, high amplitude rhythms such as delta $(0.75-4 \mathrm{~Hz})$ and theta $(4-8 \mathrm{~Hz})$ are associated with lower levels of consciousness and are typically observed during certain stages of sleep (Borbely, Hayaishi, Sejnowski, \& Altman, 2000; Destexhe \& Sejnowski, 2000). Faster low amplitude rhythms such as alpha $(8-12 \mathrm{~Hz})$, beta $(13-30 \mathrm{~Hz})$ and gamma $(30+\mathrm{Hz})$ are associated primarily with active/awake states and have been recorded during tasks involving higher-order cognitive processes such as working memory and attention (Klimesch, 1997; Klimesch, Doppelmayr, Russegger, Pachinger, \& Schwaiger, 1998; Sokolov, Pavlova, Lutzenberger, \& Birbaumer, 2004; Wrobel, 2000). Alpha-like rhythms (8-12 Hz) specific to the sensorimotor cortex are termed "mu rhythms", and are associated predominately with the processing of basic somatosensory information (Pineda, 2005). Sensorimotor mu rhythms are the primary focus of my research study and will be further discussed in Section II.

Electroencephalography (EEG) and magnetoencephalography (MEG) are non-invasive methodological approaches with exquisite temporal precision, on the order of a millisecond, which allow researchers record how the brain responds to certain cognitive 
events as they occur in real time (Ladouceur, Dahl, Birmaher, Axelson, \& Ryan, 2006). An electroencephalogram measures electrophysical cortical activity recorded at the scalp and the time course of cognitive events is usually measured by averaged event-related potentials (ERPs). The electrocortical ERP patterns produced are then used to compare and contrast how individuals process stimuli information. MEG records the minute magnetic fields that emanate from the brain thus "looks through the skull and the scalp since these tissues are transparent to magnetic fields...” (Hari, Salmelin, Makela, Salenius, \& Helle, 1997). Co-registration of MEG and magnetic resonance imaging (MRI) techniques provides superior spatial resolution to that of an electroencephalogram. Thus MEG is an optimal technique for capturing spatial and temporal information during sensory processing. MEG offers several other advantages over EEG for studying somatosensory functioning: i) the magnetic fields measured are not as influenced by low skull conductivity as electrical potentials; ii) MEG is reference-free, whereas in EEG the chosen reference type will always influence cortical effects analysis (unless average reference is used, which requires a flawless electrode reference); and iii) the magnetometers of MEG record predominantly cortical activity, which was of primary interest in my research. However, both EEG and MEG are capable of characterizing spatiotemporal neural dynamics elicited by cognitive processes, including various cortical rhythm patterns of activity (Hari et al., 1997).

MEG reflects mainly cortical activity from structures with a tangential orientation to the brain surface, so the recorded activity most likely comes from cortical structures in the walls of the gyri and sulci - which is an advantage for measuring the sensorimotor cortex. 
However, this might be considered a shortcoming of MEG, given that EEG captures activity from both radial- and tangential-oriented fibres from the whole brain. Despite the shortcomings of each of these functional neuroimaging techniques, current analysis of cortical rhythms can only be performed using EEG or MEG (Johansen-Berg \& Llyod, 2000).

\subsubsection{Attention modulation of cortical rhythms}

Attention is a cognitive process that requires the conscious allocation of processing resources towards some features in an environmental relative to the exclusion of others (Anderson, 2004; James, 1890). Selective attention is a 'top-down' process that promotes the capacity to maintain vigilant concentration to relevant sensory information, while ignoring irrelevant distracter stimuli in an environment (Womelsdorf \& Fries, 2007). Behaviourally, selective attention promotes more efficient processing, as indexed by faster reaction times, greater performance accuracy, and enhanced sensitivity to fine changes made to a stimulus (Womelsdorf \& Fries, 2007). Moreover, attention also facilitates perceptual processing of the selected sensory stimuli by modulating neural signals that convey relevant sensory information (Miller \& D’Esposito, 2005). Several studies have shown that attention-related processes can modulate electrocortical ERPs such as the P100, N100, and/or P300, as well as in various cortical rhythm bandwidths such as: alpha (8-12 Hz), beta $(13-30 \mathrm{~Hz})$, and gamma $(30+\mathrm{Hz})($ Hermann \& Knight, 2001). Thus, neurophysiological factors may help researchers to better understand human attention (Herrmann \& Knight, 2001). 
Attentional processes can influence slow and fast cortical rhythms by modifying firing rates of neural signals conveying the selected sensory information (Bauer, Oostenveld, Peeters, \& Fries, 2006; Debener, Hermann, Kranczioch, Gembris, \& Engel, 2003; Fries, Reynolds, Rorie, \& Desimone, 2001; Hsiao O’Shaughnessy, \& Johnson, 1993; Reynolds \& Chelazzi, 2004, Tiitinen et al., 1993). For instance, theta rhythms (4-8 Hz) are modulated by task monitoring and error detection (Luu, Tucker, \& Makeig, 2004). The alpha rhythm (8-12 Hz) can be delineated into two bandwidths: the lower band (8-10 Hz) is topographically widespread and associated with basic attentional tasks, while the higher band (10-12 Hz) is topographically restricted and involved with higher-order cognitive functions (Neuper \& Pfurtscheller, 2001), including attentional and memory processes (Bastiaansen, Bocker, Brunia, de Munck, \& Spekreijse, 2001; Bastiaansen \& Brunia, 2001; Jensen, Gelfand, Kounios, \& Lisman, 2002; Klimesch, Doppelmayr, Pachinger, \& Ripper, 1997; Klimesch, Doppelmayr, Russegger, Pachinger, \& Schwaiger, 1998). Since, the upper $10-12 \mathrm{~Hz}$ band is associated with cognitive functions that require highly attentive behaviour, this thesis investigated the top-down effects of selective attention to somatosensory information exclusively in this frequency range. Attention also synchronizes beta band activity $(13-30 \mathrm{~Hz})$ during the transfer of information between remote brain regions (Nikolaev, Ivanitsky, Ivanitsky, Posner, \& Abdullaev, 2001). Lastly, gamma rhythms $(30+\mathrm{Hz})$ are modulated during tasks of stimulus detection (Crick \& Koch, 1990). Evidently, the interaction between attentional processes and cortical rhythms suggests a computational role for neural activity wherein neurons representing relevant sensory information dynamically control how the brain responds to incoming sensory events (Womelsdorf \& Fries, 2007). 
To date, selective attention has been most thoroughly researched in the visual system. Both primate- and human-based studies have shown that focusing attention toward visually relevant stimuli synchronizes gamma-band oscillatory activity in the visual cortex (Tallon-Baudry, Bertrand, Henaff, Isnard, \& Fischer, 2005; Womelsdorf, Fries, Mitra, \& Desimonne, 2006). However, the auditory and somatosensory cortices also show enhanced gamma-band activity for attended versus non-attended stimuli (Bauer et al., 2006; Debener et al., 2003; Kaiser, Hertrich, Ackermann, \& Lutzenberger, 2006; Lenz, Schadow, Thaerig, Busch, \& Hermann, 2007; Sokolov et al., 2004; Tiitinen et al., 1993). For example, studies of spatial cued auditory detection report that directing attention to one ear during a selective listening task modulated auditory evoked ERPs and magnetic responses as early as 20-50ms post stimuli (Rif, Hari, Hamalainen, \& Sams, 1991; Woldorff et al., 1993). Moreover, in a recent MEG study, it was revealed that tactile selective attention to a spatially cued versus a non-cued Braille pattern enhanced gamma activity in the primary somatosensory cortex (SI) and reduced lower frequency activity in the parieto occipital areas (Bauer et al., 2006).

Selective attention is known to enhance neural activity (i.e. ERS) in various frequency bandwidths (i.e. gamma) (Bauer et al., 2006; Jensen, Kaiser, \& Lachaux, 2007) and suppress (i.e. ERD) it in others (i.e. alpha) (Bastiaanen et al., 2001; Dujardin, Bourriez, Guieu, 1995; Dujardin et al., 1993; Van Winsum, Sergent, \& Geuze, 1984). Alpha desynchrony in sensorimotor regions has been reported in attention-related tasks requiring motor preparation, attentional effort, expectancy or orientation to a relevant 
stimuli event (Barry \& Beh, 1972; Bastiaansen \& Brunia, 2001; Grunwald-Zuberbier, Grunewald, Rasche, \& Netz, 1978; Pribram \& McGuinness, 1975). Notably, an EEG study conducted 25 years ago demonstrated that the size, magnitude, and duration of alpha ERD was influenced primarily by attentional demand, such that increasing selective attention demands (i.e cognitive load) led to stronger and more prolonged alpha ERD effects (Van Winsum et al., 1984)

There is substantial evidence showing that attention modulates various cortical rhythms in humans (Batiassen et al., 2001; Bauer et al., 2006; Hermann \& Knight, 2001). However, previous research has not investigated whether attentional processes modulate cortical rhythms differently in males and females. Only recently has experimental neuroscience begun to investigate if attention-related sex differences in cortical rhythm activity exist. Intriguingly, preliminary findings suggest that neurophysiological sex differences in selective attention exist.

\subsubsection{Sex differences in selective attention}

It is widely recognized that sexual dimorphic effects in the performance of various cognitive tasks exist. In particular, visuospatial tasks such as mental rotation, spatial rotation and mathematics, have consistently shown that males outperform females. By contrast, tests of verbal ability, manual speed (i.e. finger tapping), and verbal working memory have shown that females surpass males (for full review see Kimura, 1996; Wegesin, 1998). Neuroimaging studies of mental rotation have reported sex differences in brain activation. In general, males elicit greater parietal activity while females show 
greater right prefrontal activity despite no differences in behavioural performance. Researchers hypothesize that greater parietal activation in males suggests the use of gestalt principles (object rotation/recognition) while greater right frontal activation in females suggests the use of a more top-down/serial approach to visuospatial attentional processing (Butler et al., 2006). These results suggesting that males and females may use different cognitive strategies during tasks requiring visuospatial manipulation (Butler et al., 2006; Hugdahl, Thomsen \& Ersland, 2006; Weiss et al., 2003).

Sex differences in neural activity have also been reported in studies of visual selective attention. For example, Steffensen et al., 2008, compared visual evoked potentials (VEP) and reaction times in males and females, to investigate whether neurophysiological and/or behavioural sex differences exist in selective attentional processing. The VEP has two ERP components believed to be relevant for attentional processing: 1) the P300, which is believed to be a neurophysiologic marker of attentional allocation, and target salience (Coull, 1998; Katayama \& Polich, 1998; Picton, 1992), and 2) the N400, which is thought to reflect contextual integration of potentially relevant or meaningful stimuli and is often associated with semantic processing (Finnigan, Humphreys, Dennis, Geffen, 2002; Gunter, Friederici, \& Schriefers, 2000; Hagoort, 2003; Kutas \& Federmeier, 2000). Using an object recognition task, participants were instructed to respond to randomly presented "relevant” stimuli and to ignore "irrelevant” and “standard” visual stimuli. No differences in reaction times or in the early components of the VEP were found for relevant stimuli. However, females displayed larger P300 and N400 amplitudes relative to males for the relevant stimulus. Further, only females showed a large N400 amplitude 
for irrelevant stimuli. These finding suggest that that males and females attend to visual information differently wherein females process relevant and irrelevant visual information while males only process relevant visual information. Thus, the large N400 response to irrelevant visual information found only in females may be a result of greater allocation of attentional resources to distracting stimuli.

Furthermore, in a recent EEG study, the Attention Network Test (ANT), a paradigm customized for measuring top-down attentive functions including alerting, orienting, and executive control (Fan et al., 2007), was used to investigate sex differences in VEPs. Results revealed no differential effects in behavioural performance, yet prominent sex differences in neural substrates with females displaying: i) a larger fronto-occipital second peak of the N100 ERP; ii) a larger central ERP component that was strongly correlated with stimulus saliency; and iii) greater cortical activation in the right rostral prefrontal and occipital cortices. These findings specifically emphasize the significant role that sex plays in visual attentional processing. However, for the majority, reports of neurophysiological sex differences in selective attentional processing have been based on the visual cortex.

Human and primate studies have demonstrated that the top-down effects of attention can influence cortical activity in other sensory modalities including the somatosensory cortex (Burton et al., 1999; Hsiao et al., 1993; Hyvarinen, Poranen, \& Jokinen, 1980; Iriki, Tanaka, \& Iwamura, 1996; Johansen-Berg, Christensen, Woolrich, \& Matthews, 2000; Meyer et al., 1991; Mima, Nagmine, Nakamura, \& Shibasaki, 1998). Yet, few studies 
have examined whether males and females exhibit different cortical reactivity patterns when attending to somatosensory information. Recently, studies have shown that action observation (i.e. watching another person perform a task with either the hand or foot), and/or the perception of potentially painful experiences (i.e. watching another person misuse a pair of scissors) elicits neurophysiological sex differences in sensorimotor mu rhythms localized in the somatosensory cortex wherein females showing greater mu suppression (i.e. mu ERD) relative to males (Cheng, Tzeng, Decety, Imada, \& Hsieh, 2006; Cheng, Lee, Yang, Lin, Hung, \& Decety, 2008a; Cheng, Yang, Lin, Lee, \& Decety, 2008b; Yang, Decety, Lee, Chen, \& Cheng, 2009). These results indicate that sex differences in cortical rhythm activity are not specific to the visual cortex, and imply that there is a need to investigate whether sex differences in selective attention exist for other sensory modalities such as the somatosensory cortex.

\section{SECTION II: THE SOMATOSENSORY CORTEX}

The somatosensory system comprises a variety of specialized receptors distributed over the skin and throughout the body (e.g., muscles, tendons, joints, viscera), which produce sensations such as, heat/cold, body position, pain, and touch. Somatosensory information is represented in multiple brain regions, but the primary and secondary somatosensory cortices (SI and SII) are the principal regions for tactile perception (Johansen-Berg \& Lloyd, 2000). Moreover, attentional effects have been demonstrated in both of these somatosensory regions (Burton et al., 1999; Hsiao et al., 1993; Johansen-Berg et al., 2000; Meyer et al., 1991). Research on humans has shown that SI plays the central role in early somatosensory perception (i.e. stimulus detection) and that SII is involved in the 
later stages of somatosensory processing (Mima et al., 1998). Notably, several studies have demonstrated that SI cortical activity is also modulated by higher-order processes including selective attention (Roland, Meyer, Shibasaki, Yamamoto, \& Thompson, 1982; Johansen-Berg et al., 2000; Meyer et al., 1991).

For example, evidence suggests that sensorimotor mu rhythms $(8-12 \mathrm{~Hz})$ generated in SI, are sensitive to attentional processes such as action observation (Cheng et al., 2006; Cheng et al., 2008a; Cheng et al., 2008b), expectancy of a sensorimotor event (Babiloni et al., 2004, Babiloni et al., 2005), and/or attentional alerting (Nikouline et al., 2000; Stancak et al., 2003). Although the functional role of mu activity remains unclear, these findings suggest that mu rhythms are unique in their sensitivity to tasks requiring vigilant attentional processing of somatosensory information (Pfurtscheller, 2001). Thus, mu rhythms warrant further investigation in order to gain a better understanding of how higher-order processes like attention influence cortical responses in the brain.

\subsubsection{Sensorimotor Mu Rhythms}

Mu rhythms were originally believed to only occur occasionally and in a limited percentage of the general population (Koshino \& Isaki, 1986). Technical advances in neuroimaging methods (such as the development of MEG) have demonstrated that mu rhythms are prominent oscillations that can be found in infancy (Stroganova, Orekhova, \& Posikera, 1999), and increase in strength and frequency with cortical maturation (Marshall, Bar-Haim, \& Fox, 2002; Stroganova, et al., 1999). MEG’s sensitivity to neural 
activation in the fissural cortex makes it an optimal technique to measure mu rhythms which are buried deep within the rolandic region of the brain (Hari, 2006).

Initially, mu rhythms were thought to reflect a cortical idling state since synchronization of the mu rhythm was found in the absence of processing somatosensory information and in the absence of motor output (Adrian \& Matthews, 1935; Chase \& Harper, 1971; Mulholland, 1995; Pfurtscheller, Stancak, \& Neuper, 1996). For example, enhanced mu activity in the primary hand area during visual processing and/or during foot movement has been reported (Pfurtscheller et al., 1996). It is speculated that mu enhancement occurs during unrelated sensorimotor tasks because neurons devoted to processing hand-related information are not required, thus exhibit a 'nil-working' state, comparable to the occipital alpha rhythm and visual processing (Kuhlman, 1978).

Despite alpha-like oscillatory characteristics, mu rhythms are distinct in several ways: i) they exhibit a more anterior source localization compared to the more posterior localization of the classic alpha oscillations (Pineda, 2005), and ii) they are asymmetrical in frequency and power, and do not exhibit bilateral coherence, dissimilar from classic alpha rhythms (Steriade \& Llinas, 1988). Moreover, mu rhythms are only minimally influenced by visual stimulation that is irrelevant to the sensorimotor system (i.e. visual presentation of a dot moving randomly across the screen) unlike classic alpha rhythms, and instead are primarily suppressed by voluntary movement, motor imagery (Muthukumaraswamy \& Johnson; 2004; Muthukumaraswamy, Johnson, \& McNair, 2004), action or pain observation (Cheng et al., 2006; Cheng et al., 2008a; Cheng et al., 
2008b; Yang et al., 2009),and/or somatosensory stimulation (Neuper, Wortz, \& Pfurtscheller, 2006; Nikouline et al., 2000; Pfurtscheller \& Neuper, 1994).

Although the exact functional role of mu rhythms remains unknown, substantial progress in elucidating the functional significance of these rhythms has been made. It is now known that mu rhythms are not only involved in basic somatosensory processing, but are also sensitive to higher-order attentional processing such as action observation and mimicry (Cheng et al., 2006; Cheng et al., 2008a; Cheng et al., 2008b; Virji-Babul et al., 2008), as well as affective influences such as the perception of pain in others (Yang et al. 2009). Moreover, a recent study found that mu rhythms can be further delineated into two attention-related subtypes: a lower somatotopically non-specific $8-10 \mathrm{~Hz}$ band associated with basic attentional processing of somatosensory information, and an upper $10-12 \mathrm{~Hz}$ somatotopically specific band which is elicited in tasks requiring selective attention to a motor subsystem (Pfurtscheller, 2001).

During movement preparation or tactile stimulation, mu rhythms are suppressed and show a characteristic pattern of ERD, indicating cortical activation of the sensorimotor region and thus active processing of the somatosensory stimuli (Neuper et al., 2006; Nikouline et al., 2000; Pfurtscheller \& Neuper, 1994; Pfurtscheller, Pregenzer, \& Neuper, 1994). More specifically, an early and transient suppression of mu activity (ERD) occurs which is followed by a subsequent burst of synchronous activity (ERS) occurring bilaterally over central regions post movement. These changes can be observed bilaterally but are typically stronger in the contralateral hemisphere (Devos et al., 2006; 
Nikouline et al., 2000; Salmelin \& Hari, 1994; Stancak \& Pfurtscheller, 1996). Various experiments involving somatosensory processing and motor performance have described task-related ERD, followed by an increase in power above baseline levels (event-related synchrony; ERS) (Shishida, Hashizume, Onoda, Okamoto, Yamawaki, 2006; Toro et al., 1994). This well documented event-related patterning suggests that somatosensory events (whether evoked or voluntary) provoke early activation in SI areas indicative of rapid processing of the sensory stimuli (Pfurtscheller, 2001), while execution of the movement causes bilateral deactivation in (or alternatively, a resetting of) sensorimotor areas (Huttunen, Komssi, \& Lauronen, 2006; Lin \& Forss, 2002; Sutherland \& Tang, 2006).

Recent evidence indicates that mu rhythms are also suppressed (ERD) by various higher order cognitive functions involving voluntary control of attention such as: movement imitation (Virji-Babul et al., 2008), movement observation (Muthukumaraswamy et al., 2004), the mental imagery of movement (Neuper et al., 2006; Pfurtscheller, Brunner, Schlogl, \& Lopes da Silva, 2006), motor sequence learning (Zhuang et al., 1997), the attentional orienting to a sensorimotor cue (Bender, Resch, Weisbrod, \& Oelkers-Ax, 2004).

Researchers are now suggesting that mu rhythms play a critical role in the human mirror neuron system which is thought to be responsible for linking perception to action or otherwise stated as "the transformation of perceiving into doing”' (Pineda, 2005). Mirror neurons are a distinct class of neurons with visuomotor properties originally discovered in ventral premotor and posterior parietal cortices of monkeys and activate during 
execution of observation of goal-oriented actions (Fadiga \& Craighero, 2004; Gallese, Fadiga, Fogassi, \& Rizzolatti, 1996; Rizzolatti, Fadiga, Fogassi, \& Gallese, 1996).

Recent research has found sex differences in mu reactivity of the mirror neuron system of humans (localized in SI) with females eliciting stronger mu suppression compared to males during simultaneous presentation of median nerve stimulation (MNS) and videotaped hand actions (Cheng et al., 2008a). Suppression of mu reactivity is thought to reflect “downstream modulation of primary sensorimotor areas by mirror neuron activity (Pineda, 2005). These findings suggest that mu rhythmic activity is best examined in primary rather than secondary sensorimotor areas. However, action observation requires a high level of attentional demand for action comprehension to occur thus sex differences in mu reactivity may reflect sex differences in the attentional processing of relevant somatosensory information. The primary aim of my research was to address this possibility by investigating whether selective attention to somatosensory information modulates mu reactivity (localized in SI) differently in males and females.

\subsubsection{Mu Rhythms and the empathetic response hypothesis}

Several hypotheses have been made regarding the functional significance of mu rhythms. Indeed, both affective and cognitive influences such as the level and quality of attention, and motor immobility can influence the occurrence and maintenance of mu rhythms (Pineda, 2005). However, the most provocative suggestion is that mu rhythms reflect activity in visuo-, audio-, and sensorimotor mirror neurons in frontal and parietal networks crucial for linking perception and actions, or otherwise stated as the transformation of "seeing” and "hearing” into "doing”. Support for this hypothesis is 
generated from the findings that mu rhythms are modulated by observation of goaldirected actions, motor and cognitive imagery, as well as are generated in sensorimotor areas akin to mirror neuron activity (see Pineda, 2005 for full review).

Moreover, mu rhythms may serve a purposeful role in pain perception and/or empathetic mimicry. Empathetic mimicry can be defined as the ability to copy another person's abilities (i.e. emotions or actions) and voluntarily reproduce them at will. For example, a recent EEG study reported that the perception of pain in another individual suppressed mu activity localized in the primary somatosensory cortex (SI) of healthy adults (Cheng et al., 2008b). Subjects received left median nerve stimulation while watching static depictions of body limbs in potentially painful and non-painful circumstances. The decision to use left MNS is significant since studies have shown that the right hemisphere may be more sensitive to empathetic situations than the left hemisphere (Perry et al, 2001, Ruby \& Decety, 2003; Ruby \& Decety, 2004), however these results have not been consistently reported. Results showed that both visual stimuli suppressed the $\sim 10 \mathrm{~Hz} \mathrm{mu}$ response but the potentially painful stimuli generated significantly stronger mu suppression relative to the non-painful stimuli. Additionally, mu suppression was positively correlated with the perspective taking subscale of the interpersonal reactivity index, a self-report measure of dispositional empathy. Based on this finding, the researchers hypothesized that mu rhythms may be a potential biomarker of empathetic mimicry (Cheng et al., 2008b). 
Empathy is particularly important for social interaction and moral development as it enables an observer the ability to interpret and comprehend other people's feelings without confusion between self and other (Decety \& Batson, 2007; Decety \& Jackson, 2004; Decety \& Lamm, 2006). While it remains a matter of debate, much psychological research suggests that sex differences in empathy exist. For instance, females tend to be more accurate when interpreting others' facial expressions and body actions, and typically outperform males in tests of emotional recognition (Baron-Cohen, Knickmeyer, \& Belmonte, 2005; Geary, 1998; Hall, 1984; McClure, 2000). Empathetic responses rely on unconcious emotional mimicry for the proper translation of affective sharing between self and other (Decety \& Batson, 2007; Decety \& Jackson, 2004; Decety \& Lamm, 2006). This translation relies on the perception-action coupling of the human mirror neuron system, and automatically activates neural responses in the sensorimotor cortex in the observer (Preston \& de Waal, 2002). Thus, research studies examining whether males and females manifest differential mu reactivity during tasks involving actionobservation/comprehension have been conducted in an attempt to further elucidate the purported sex differences in empathy.

\subsubsection{Sex differences in mu modulation}

In the first investigation of potential sex differences in mu rhythms, Cheng et al., 2006 instructed participants to watch visual presentations of either a male hand with androgynous characteristics manipulating an object (i.e. relevant information for the mirror neuron system and action comprehension), or a randomly moving dot (nonrelevant information for the mirror neuron system). Results indicated that females 
showed greater mu suppression when watching hand actions versus the moving dot whereas males showed the opposite effect (i.e. greater mu suppression when watching the moving dot versus the hand actions). The authors concluded that sex strategies might have been used to process moving dot stimuli, wherein males were more likely to categorize the moving dot as an 'object' (Cheng et al., 2006). This study provided the initial support for the working hypothesis that mu rhythms play a key role in unveiling sex differences in action comprehension and the human mirror neuron system. However, it was cautioned that the male hand stimuli used may have been partially responsible for eliciting the greater mu suppression in females due to the opposite-sex effect, thus may have confounded these results.

To account for this possibility, Cheng and colleagues conducted another study using EEG, but this time, the hand actions presented included androgynous, male, and female characteristics. Additionally, a self-report scale of empathy was utilized to investigate if mu reactivity correlated with dispositional measures in each group. Results replicated the previous study in that females showed significantly greater mu suppression than males when watching hand actions which was not biased by the sex of the perceived hand used (i.e. no significant differential mu suppression between the perceived same-sex and opposite-sex was revealed). However, observation of the moving dot elicited minimal mu suppressions in both males and females, although to a slightly greater extent in males. Notably, mu suppression for hand stimuli was positively correlated with the personal distress subscale and negatively correlated with the systemizing quotient of the interpersonal reactivity index used. Thus, observation of hand actions performed by 
another individual, elicited greater mu suppression in females who also scored higher on measures of affective response to others and lower in systemizing ability while males showed the opposite effect (i.e. diminished mu ERD for hand actions which correlated with lower scores on affective response and higher scores on systemizing ability). Based on this correlational finding, it was speculated that sex differences in the mu response might reflect sex differences in empathetic mimicry (Cheng et al., 2008a).

A recent fMRI study examining sex differences in right hemisphere activation and empathy demonstrated correlation between empathy questionnaire scores and right hemisphere activation in females only (Rueckert \& Naybar, 2008). Researchers of this study suggested that sex differences in neural activation may be associated with sex differences in empathy. To test whether mu rhythms mimic sex differences in empathy, Yang et al., 2009 used MEG to investigate mu responses elicited in males versus females during pain perception. Using a similar paradigm to Cheng et al., 2006, participants viewed static pictures of potentially painful and non-painful experiences, as well as filled out a self-report dispositional measure of empathy. Results showed greater mu suppression overall in females relative to males during painful and non-painful actions. Moreover, the pain-related mu suppression in females was positively correlated with their scores on the personal distress subscale of the interpersonal reactivity subscale while no such effect was found for males. Moreover, no significant correlations were found for non-painful mu suppression or for dispositional measure of empathy which implies that the greater pain-related mu suppression expressed in females may reflect a stronger sensitivity to pain perception. These findings are provocative and have provided a 
fundamental link between mu rhythms, action comprehension, the human mirror neuron system, as well as offer a possible neurophysiological explanation for the well-known sex differences in empathy. However, females also showed greater mu suppression relative to males during observation of non-painful hand stimuli (i.e. presentation of a dot moving randomly across a screen). Based on this result, it seems unlikely that mu rhythms solely reflect sex differences in empathy mimicry. Moreover, a recent study conducted by our research groups found differential mu reactivity between individuals with ADHD and healthy controls during a passive attentional task using non-painful tactile stimulation (Dockstader, Gaetz, Cheyne, \& Tannock, 2009), which suggests that the mu response may be sensitive to attention-related processes in general. Thus, the sex differences found during action observation and pain perception may instead reflect differences in how males and females attend to sensory information.

\subsubsection{Advantages of using MEG and MNS to study somatosensory processing}

Somatosensory processing is optimally characterized by MEG in combination with median nerve stimulation (MNS) (Johansen-Berg \& Lloyd, 2000). MEG’s high temporal resolution, combined with novel source reconstruction techniques capable of millimetre spatial resolution, makes MEG an optimal technique for capturing spatial and temporal information during sensory processing for which the time scale is on the order of milliseconds (Ioannides, 2009). MNS is a technique in which a series of non-painful, brief electrical pulses (e.g., 0.2 ms duration) are presented just above motor threshold (eliciting a small, passive, thumb twitch) and applied transcutaneously to the median 
nerve at the wrist. This technique has been used to capture changes in cortical oscillations in response to somatosensory stimuli to determine the integrity of somatosensory cortical function (Huttunen et al., 2006; Lin \& Forss, 2002; Sutherland \& Tang, 2006; Womelsdorf \& Fries, 2007). MNS is a limited technique in that it is a nonnatural stimulus (i.e. it is not a somatosensory event that is encountered frequently throughout one's life time), and is susceptible to inter-person variability with regard to the perceived level of discomfort experienced. However, I chose to use MNS in my study for several reasons, namely: i) the mu rhythm is transiently increased by MNS allowing the ability to quantify task-related changes in source power, ii) MNS generates a precise and robust SI response that is highly reproducible and well documented, and iv) MNS produces less variability in SI reactivity relative to spontaneously occurring rhythmic activity (Salenius, Portin, Kajola, Salmelin, Hari, 1997a; Salenius, Schnitzer, Salmelin, Jousmaki, Hari, 1997b).

MEG studies of the human somatosensory system using MNS have shown that only the contralateral primary somatosensory cortex (SI) responds predominately to unilateral tactile information whereas bilateral secondary somatosensory cortices (SII) show activity in response to unilateral stimulation (Lin \& Forss, 2002; Forss, Salmelin, \& Hari, 1994). The earliest somatosensory activity occurs at approximately 20 ms poststimulation in SI just caudal to the central sulcus in the corresponding topographical location (i.e. hand area $3 \mathrm{~b}$ in the hemisphere contralateral to the moved body part). Subsequent somatosensory activation occurs in bilaterally in SII located in the dorsal regions of the lateral sulci (Hari et al., 1984; Forss et al., 1994; Tiihonen, Hari, \& 
Hamalainen, 1989). Overall, basic or complex sensory processing requires a dynamic interaction between groups of neurons oscillating at particular frequencies and differing degrees of coupling. As a result, source activity in SI and SII, following median nerve stimulation, demonstrate both alpha and beta cortical rhythms (Della Penna et al., 2004).

Overall, these relatively rapid changes in cortical oscillations during sensory processing reflect both low-level processing of the incoming sensory information and cognitive and attention-related influences on sensory networks underlying normal perceptual functions (Womelsdorf \& Fries, 2007; Womelsdorf et al., 2007).

\section{SECTION III: RATIONALE FOR MY STUDY}

The advancement of non-invasive temporally precise neuroimaging techniques such as MEG and EEG have allowed cognitive neuroscientists to examine how certain higherorder cognitive events such as attention induce neurophysiological changes in cortical rhythm activity. Attention can influence the neural synchrony (i.e. the temporal relation between neurons) of cortical rhythms in two ways: i) event-related synchrony (ERS), wherein the firing rate of multiple neurons is correlated, and ii) event-related desynchrony (ERD), where neurons fire in a randomized fashion. ERS and ERD phenomenon reflect increases and decreases in source power relative to baseline states (Neuper \& Pfurtscheller, 2001) and indicate cortical deactivation or activation of a sensory area, respectively. 
The visual system is recognized as the most extensively researched sensory modality. Numerous studies have shown that the neurophysiological representations such as the VEP and cortical rhythms (i.e. alpha, gamma) are sensitive to the top-down effects of selective attention (Hermann \& Knight, 2001; Katayama \& Polich, 1998). However, attentional effects have also been reported in other sensory modalities such as the somatosensory cortex. Thus, there is considerable need to investigate whether selective attention alters neuronal responses in all sensory modalities, and if so how?

Moreover, recent evidence has shown attention-related sex differences in neural activity occur in the visual cortex (Steffensen et al., 2008), and to a somewhat lesser extent, in the somatosensory cortex (Cheng et al., 2006; Cheng et al., 2008a, Yang et al., 2009). Studies of visuospatial attention (Butler et al., 2006; Gron, Wunderlich, Spitzer, Tomczak, \& Riepe, 2000; Jordan, Wustenberg, Heinze, Peters, \& Jancke, 2002) and action observation (Cheng et al., 2006; Cheng et al., 2008, Cheng et al., 2008b) have suggested that sex differences in neural activity patterns may reflect sex-specific cognitive strategies. However, in contrast to the visuospatial reports of sex differences in selective attention, studies reporting sex differences in somatosensory cortices have not explicitly requested participants to vigilantly focus their attention and instead have been based on passive observation of another person's actions (Cheng et al., 2006; Cheng et al., 2008a, Cheng et al., 2008b; Yang et al., 2009). Thus, how males and females selectively process somatosensory stimuli remains unknown. 


\subsection{Aims and Hypothesis}

The primary aim of my study was to experimentally manipulate attention to somatosensory events in order to determine whether sex differences in mu reactivity are a result of attention-related sex differences in somatosensory processing. My hypotheses were that: i) mu suppression (i.e. ERD) would be strongest when attention was directed to relevant somatosensory information (i.e. MNS) and ii) there would be attention-related sex differences in the mu response with females showing greater mu reactivity than males. 


\section{PART II \\ SEX DIFFERENCES IN SENSORIMOTOR MU RHYTHMS DURING ATTENTIONAL PROCESSING}




\subsection{Introduction}

Attention can be directed either voluntarily to task-relevant sensory information (i.e. topdown approach), or automatically by salient sensory stimuli (i.e. bottom-up approach). Behaviourally, selective attention promotes more efficient information processing, as indexed by faster reaction times, greater performance accuracy, and enhanced sensitivity to fine changes made to a stimulus (Womelsdorf \& Fries, 2007). Moreover, animal and human studies have shown that selective attention to somatosensory information can modulate the response of neural populations in sensorimotor regions of the brain (Johansen-Berg \& Llyod, 2000; Mima et al., 1998). Recently, studies have shown sex differences exist in sensorimotor mu rhythms (cortical oscillations of 8-12Hz) during the observation of another person's actions (Cheng et al., 2006; Cheng et al., 2008). These findings suggest that mu rhythms may be an important neurophysiological marker useful for unveiling sex-specific neural responses elicited during attention-related somatosensory processing. However, it remains unclear how males and females selectively process somatosensory information.

Mu rhythms are associated primarily with processing of basic somatosensory information (Pineda, 2005), and are generally more robust in the hemisphere contralateral to the involved body part (Devos et al., 2006; Nikouline et al., 2000; Samelin \& Hari, 1994; Stancak \& Pfurtscheller, 1996; Stancak et al., 2003). During somatosensory stimulation and/or during motor preparation and execution, these rhythms exhibit a characteristic pattern of reactivity that is reliably reproduced (Neuper, et al., 2006; Nikouline et al., 2000; Pfurtscheller \& Neuper, 1994). More specifically, immediately following sensory 
stimulation mu rhythms are suppressed (i.e. event-related desychrony; ERD) followed by a gradual return to their baseline state, and are sometimes proceeded by minimal synchronization effect (i.e. event-related synchrony; ERS) (Szurhaj et al., 2003). ERD and ERS phenomenon refer to increases and decreases in neural activity below (ERD) or above (ERS) baseline levels, and are believed to reflect sensorimotor activation and inhibition, respectively (Lopes da Silva \& Pfurtscheller, 1999; Salmelin \& Hari, 1994).

Mu rhythms are also suppressed by higher-order attention-related demands such as cognitive and affective influences (Pineda, 2005), action observation (Cheng et al., 2006; Cheng et al., 2008a), and imitation (Virji-Babul et al., 2008), stimulus expectancy (Babiloni et al., 2004; Babiloni et al., 2006a; Babiloni et al., 2006b), attentional alerting to somatosensory input (Nikouline et al., 2000), and pain perception (Cheng et al., 2008b; Yang et al., 2009). Moreover, evidence has shown that two types of attention-related mu rhythms exist: i) a lower somatotopically non-specific band (8-10Hz) elicited during general tasks demands when vigilant attention is not explicitly required, and ii) an upper somatotopically specific band (10-12 Hz) which is observed during highly attentive behaviour (Montaron, Bouyer \& Rougeul-Buser, 1979; Pfurtscheller, Neuper, \& Krauz, 2000). It is speculated that suppression of lower frequency mu rhythms reflects general attentional processing, while upper frequency mu suppression reflects selective attention to a motor subsystem (Pfurtscheller et al., 2000). Thus, the specific aim of this study was to determine whether males and females exhibit different mu reactivity patterns specifically in the $10-12 \mathrm{~Hz}$ range when attention is directed to somatosensory information. 
Studies have shown that sex is a critical variable to consider when investigating the ascribed functional roles of mu rhythms. For instance, a recent study found sex differences in mu reactivity while observing pictures of potentially painful experiences happening to another person: females showed greater suppression of mu reactivity compared to males, suggesting a greater empathetic response in females (Yang et al., 2009). Accordingly, mu rhythms have been proposed as a potential biomarker for unveiling sex differences in empathy mimicry (Yang et al., 2009). However, evidence of greater mu suppression in females during observation of non-painful limb actions (i.e. hand manipulation of a 3-dimensional object and/or using a pair of scissors) (Cheng et al., 2006; Yang et al., 2009) indicates that sex differences in the mu response are not specific to empathetic situations. Alternatively, we speculate that sex differences in sensory processing might be attributable to differences in attention - a mechanism by which sensory events are selected for further processing. More specifically, we propose that top-down control of attention towards and away from somatosensory information might elicit sex differences in the $10-12 \mathrm{~Hz}$ mu bandwidth due to its sensitivity to higherorder attentional functions.

Growing evidence suggests that there are measurable differences in how males and females selectively direct their attention to sensory stimuli (Neuhaus et al., 2009; Ruytiens et al., 2007; Steffensen et al., 2008). For example, a recent electroencephalography (EEG) study found sex differences of the visual evoked potential during a self-directed attention task, which suggested that females may process visual 
information in a more effortful manner than males by allocating more attentional resources to distracting stimuli (Steffensen et al., 2008). However, the neural underpinnings of sex differences in self-directed attention to somatosensory information remain unclear.

Accordingly, our primary objective was to determine whether males and females differ in top-down control of attention to sensory stimuli. We used magnetoencephalography (MEG), in combination with an established paradigm (median nerve stimulation, MNS) to investigate whether self-directed attention towards and away from non-painful somatosensory information has a differential effect on the sensorimotor mu response in males and females. MEG is a safe and non-invasive neuroimaging technique that records and amplifies the small magnetic fields generated by electrical currents in neurons, in real time and with exquisite temporal precision (ms-level) that is required for capturing oscillatory brain patterns underlying sensory processes (Ioannides, 2009).

We used two self-directed attention conditions, which were identical in terms of the stimuli presented and differed only in terms of the instructions given to participants. Visual stimuli consisted of a silent video of a popular cartoon, which was presented on a monitor screen within the participant's field of view. Somatosensory stimuli consisted of a series of non-painful electrical pulses presented in predictable trains of four stimuli (interstimulus interval of $2 \mathrm{~s}$ ) followed by a $4 \mathrm{~s}$ interval between trains when no stimulus was delivered. Conditions were presented in counterbalanced order and consisted of two 6-minute trials comprised of approximately 135 MNS events and 35 4-s MNS break 
intervals. Participants were instructed to direct their attention to the video and ignore the MNS (Attend away from MNS), or to direct their attention to the MNS and ignore the video (Attend to MNS). However, to ensure equivalent exposure to both visual and electrical stimuli in each condition, we instructed participants to fixate on the video, even when attending to the MNS. Participants were also asked to maintain a mental count of specified task-relevant events (i.e. 4 s MNS break intervals; specified video incidents) and to report their count after each condition, to provide a check on compliance with instructional set, as well as an index of performance. Following the experiment, participants completed a visual analog scale to indicate their perception of the level of attentional demand that was required for each condition.

We hypothesized that: i) mu suppression would be strongest when attending to the MNS, as a result of self-directed attention to somatosensory information; and ii) there would be attention-related sex differences in the mu response with females showing greater mu reactivity than males.

\subsection{Results}

\section{Females are physiologically more responsive to somatosensory stimuli}

For both males and females, the source of peak activation was localized to the primary somatosensory (SI) region in the hemisphere contralateral to electrical stimulation (see Figures 1 A, B). There were no sex differences in the intensity of the electrical current required to achieve motor thresholds (mean current intensity for females $=4.6$ micro Amps $(\mu \mathrm{A})$ versus males $=4.7 \mu \mathrm{A})$. 
By contrast, analysis of the N20 response to the first stimulus in a train revealed marked sex differences in the magnitude of the neuronal physiological response under both directed-attention conditions. The N20 is a dipole peak value localized to the SI regions that occurs approximately 20 ms following electrical stimulation. SI dipole values were greater in females than in males: mean dipole moment $=18.8$ nanoAmpere-meters (nAm) for females, and 12.4 nAm for males, when attention was directed towards MNS; and $17.5 \mathrm{nAm}$ for females and $12.4 \mathrm{nAm}$ for males, when attention was directed away from MNS towards the video. However, the observed sex differences did not reach conventional significance levels in a 2 X 2 repeated measures ANOVA with Condition (Attend to MNS, Attend away from MNS) as the within subject variable and Sex as the between subject variable(main effect for sex, $F[1,16]=3.29, p=0.08$, partial $\eta^{2}=0.17$ ). Moreover, neither the main effect of condition $\left(F[1,16]=0.29, p=0.6\right.$, partial $\left.\eta^{2}=0.018\right)$, nor the condition $\mathrm{x}$ sex interaction was significant $\left(\mathrm{F}[1,16]=0.26, \mathrm{p}=0.62\right.$, partial $\eta^{2}=$ 0.02).

\section{Females and males both show the typical overall pattern of somatosensory activation}

For each participant, we created Time Frequency Response plots (TFRs) to analyze specific time-frequency parameters of the SI neural response elicited in each condition. As evident in the grand averaged sex-specific TFRs (see Figure 2), both males and females show the characteristic somatosensory response in both directed-attention conditions. In both groups, electrical stimulation evoked a broadband transient burst of neural synchrony followed by the typical mu response pattern (albeit to a somewhat 
lesser extent in males): initial suppression of mu rhythms (ERD) occurring approximately $0.1 \mathrm{~s}$ following stimulation followed by a beta rebound effect at $0.5 \mathrm{~s}$ (i.e. a subsequent ERS effect occurring $\sim 20 \mathrm{~Hz}$ following the initial $\sim 10 \mathrm{~Hz}$ ERD) (Neuper \& Pfurtscheller, 2001; Pfurtscheller, Neuper, Brunner, \& Lopes da Silva, 2005), in the hemisphere contralateral (i.e. left) to the stimulation site (i.e. MNS applied to the right wrist).

\section{Females show prolonged somatosensory activation when attending to MNS}

To investigate sex differences in the SI neural response during each directed-attention condition, we averaged specific time-frequency windows of mu reactivity. As hypothesized, the most robust sex differences for our attentional paradigm were found in the $10-12 \mathrm{~Hz}$ mu bandwidth, thus we chose to restrict all of our statistical analysis to this frequency range. Two separate temporal windows were chosen to monitor mu reactivity throughout the entire duration of each task. We examined mu reactivity to the first three stimuli in a train separately from reactivity following the forth stimulus to permit more rigorous measurement of the entire extent of the mu response elicited during each attentional paradigm.

We analyzed mu activity generated $1.2 \mathrm{~s}$ after stimulation for the first three stimulus events in the train (and thus after the beta rebound effect, (Figure 3) to observe the sexspecific mu responses elicited after each stimulus event prior to the attentional count. Next, we analyzed mu activity following the fourth stimulus in a train (Figure 5) for several reasons: 1) to permit a more precise and comprehensive analysis of mu reactivity, since it was not interrupted by responses to the next stimulus in a train (Dockstader et al., 
2009); and 2) to investigate sex-related mu reactivity during the attentional count period (i.e., the MNS event-count would be updated during the $4 \mathrm{~s}$ MNS temporal break between trains in the Attend to MNS task).

As shown in Figure 3, when attention was directed toward the somatosensory events, females showed unremitting suppression of the mu response (i.e., mu ERD) for the entire duration of the trial, indicating prolonged activation of the somatosensory area (Lopes da Silva \& Pfurtscheller, 1999; Neuper \& Pfurtscheller, 2001) and perhaps more vigilant processing of the sensory stimulus. By contrast, males showed a brief increase in mu power above baseline (i.e., mu ERS), reflecting sensorimotor inhibition suggestive of rapid disengagement from sensory processing. However, no sex differences were evident in mu reactivity when directing attention away from somatosensory events and towards a visual stimulus: both showed brief mu suppression immediately following each stimulus event. Statistical analysis revealed a marginally significant Condition $\mathrm{x}$ Sex interaction $\left(F[1,18]=4.125, p=0.057\right.$, partial $\left.\eta^{2}=0.19\right)$ wherein females expressed greater mu suppression (mean mu reactivity $=-0.5, \mathrm{SE}=0.35$ ) than males (mean mu reactivity= 0.14, SE $=0.23$ ) in the Attend to MNS task (see Figure 4). . Neither the main effect for condition $\left(F[1,18]=0.95, p=0.34\right.$, partial $\left.\eta^{2}=0.05\right)$, or $\operatorname{sex}(F[1,18]=1.40, p=0.25$, partial $\left.\eta^{2}=0.07\right)$ was significant. This pattern of findings suggests that females may show a sexspecific neural profile when voluntarily directing their attention towards somatosensory stimuli. 
The most robust sex differences in neural indices of attention modulation were found for mu reactivity beginning approximately $1 \mathrm{~s}$ following the last stimulus in a train (Figure 5). In the Attend to MNS condition, females showed a strong and continuous mu suppression that persisted for almost the entire duration of the $4 \mathrm{~s}$ MNS break interval. By contrast, there was no evidence of this attention-related mu modulatory effect in males. However, this sex-specific mu ERD effect did not occur when females directed their attention away from MNS and towards the video. Statistical results showed a significant Condition $x$ Sex interaction $\left(F[1,18]=5.54, p=0.03\right.$, partial $\left.\eta^{2}=0.24\right)$ (Figure 6). There were no significant main effects found for condition $\left(F[1,18]=2.64, p=0.12\right.$, partial $\eta^{2}=$ $0.13)$, or for $\operatorname{sex}\left(F[1,18]=1.34, p=0.26\right.$, partial $\left.\eta^{2}=0.07\right)$. Post hoc tests indicated sex differences in mu reactivity elicited in the Attend to MNS task, with females displaying stronger mu suppression $($ Mean $=-0.55, \mathrm{SE}=0.28)$ versus males $($ Mean $=0.16, \mathrm{SE}=0.1$ ), as well as a within-group difference for females based on condition with Attend to MNS eliciting significantly stronger mu suppression (Mean $=-0.55, \mathrm{SE}=0.28$ ) versus Attend away from MNS (Mean =-0.03, SE=0.24).

\section{No sex differences in behavioural performance}

Self-Reported count of 'attended events'. Counting errors were computed as a deviation score (difference between the participant's count and the confirmed count) and reported as a positive value regardless of whether the count was an over- or under-estimation. Participants were required to count approximately 35 MNS events (Attend to MNS mean error score $=0.95, \mathrm{SE}=0.23$ ) and 12 cartoon events (Attend away from MNS mean error score $=0.95, \mathrm{SE}=0.23$ ). A $2 \times 2$ repeated measures ANOVA with Condition (Attend to 
MNS, Attend away from MNS) as the within subject variable and Sex as the between subject variable revealed no significant main effects for condition $(F[1,18]=0.0, p=1.0$, partial $\left.\eta^{2}=0.00\right)$, or sex $\left(F[1,18]=1.49, p=.24\right.$, partial $\left.\eta^{2}=0.08\right)$, nor was there a significant condition $x$ sex interaction $\left(F[1,18]=1.34, p=0.26\right.$, partial $\left.\eta^{2}=0.07\right)$.

Ratings of Perceived Attentional Demand. We analyzed participants' scores on a 10 $\mathrm{cm}$ visual analog scale for each condition to assess if their perceived level of attentional demand varied by condition (as predicted) or sex, or both factors. Consistent with our predictions, there was a significant main effect of condition $(F[1,18]=44.44, p=0.000$, partial $\eta^{2}=0.71$ ) with Attend to MNS condition being rated as more demanding of attention than the Attend away from MNS condition (Attend to MNS mean $=6.35$, $\mathrm{SE}=0.54$, Attend away from $\mathrm{MNS}=1.94, \mathrm{SE}=0.36$ ). Neither the main effect for sex $\left(\mathrm{F}[1,18]=.04, \mathrm{p}=.84, \eta^{2}=0.00\right)$ nor the interaction between condition and sex was significant, $\left(\mathrm{F}[1,18]=1.81, \mathrm{p}=0.20\right.$, partial $\left.\eta^{2}=0.09\right)$.

\subsection{Discussion}

This study investigated the effect of attention directed toward and away from somatosensory stimuli on mu rhythms in the contralateral primary somatosensory cortex of healthy adults. Findings included: i) greater mu suppression in females when selectively attending to somatosensory stimuli, ii) attentional modulation of the mu response elicited only in females; and iii) an enhanced (although non-significant) physiological response to MNS in females. These findings suggest that there may be sex differences in how attention to a somatosensory stimulus influences the sensorimotor mu 
rhythm. A possible explanation for these findings may be that females may process sensory information more vigilantly that males, thus elicit greater mu suppression in tasks that require extra attentional effort. However, the sex effects in mu reactivity found in this study will need to be replicated before this speculation can be confirmed.

Studies of visuospatial processing have suggested that males and females may utilize different cognitive strategies (Clements-Stephens, Rimrodt, \& Cutting, 2009; Jordan et al., 2002). Females tend to use a more effortful approach, involving continual "online” processing of salient stimuli in order to keep track of relevant information (Gron et al., 2000). Males tend to apply 'simpler' principles, based on grouping or categorizing multiple geometric cues (Gron et al., 2000; Jordan et al., 2002). Behaviourally, the attend to MNS condition was rated as the most attentionally demanding by both males and females, and both sexes performed at an equally high accuracy rate. However, in this condition, only females expressed enhanced and prolonged mu suppression, indicating long-lasting sensorimotor activation throughout the entire task. These findings suggest that females, but not males, may vigilantly process somatosensory stimuli perhaps due to a more effortful top-down approach. By comparison, males showed mu synchronization after every stimulus event reflective of sensorimotor inactivation, yet this rapid disengagement from somatosensory processing did not compromise their performance (i.e. both males and females maintained an accurate count of the task specific events). It is hypothesized that males tend to use a categorical approach to stimuli during spatial navigation tasks (Jordan et al., 2002) which may lessen the cognitive load placed on attentional resources. Thus, it is plausible, that males might have required a more 
attentionally demanding load of sensory information in order to elicit the mu suppression effect observed in females. However, we acknowledge that a direct comparison between sex-specific cognitive strategies used to perform highly complex visuospatial tasks cannot be applied directly to sex differences in the processing of basic somatosensory information. Furthermore, it is important to note that we do not suggest here that there is a causal relation between the sex differences in mu reactivity and sex-specific attentional strategies that may have been used to perform either task. Instead, the theory that males and females use different attentional strategies for visuospatial tasks should be used as a contextual reference with respect to the sex differences found in our study.

Lastly, we report a non-significant trend in the neurophysiological response to nonpainful MNS with females showing a greater neural response overall. Females have shown greater neural sensitivity during both resting states (Barry, Clarke, McCarthy, \& Seligowitz, 2006; Barry et al., 2005) and physiological stimulation (Kakigi \& Shibasaki, 1992). Studies of experimentally-induced acute pain show that females exhibit greater sensitivity to painful stimuli (Fillingim \& Maixnerm, 1995), exhibit lower pain thresholds, lower pain tolerance, and increased unpleasantness ratings to noxious stimuli (Lund, Lundenberg, \& Kowalski, 2005; Maffiuletti, Herrero, Jubeau, Impellizzeri, \& Bizzini, 2008) as well as suffer more from chronic pain conditions such as fibromyalgia and temporomandibular joint dysfunction (Fillingim \& Maixnerm, 1995; Paller, Campbell, Edwards, \& Dobs, 2009; Wiesenfeld-Hallin, 2005). Attention-related sex differences in pain processing have also been reported, wherein male tolerance to painful stimuli modulated according to the type of attentional strategy used (i.e. attentional focus 
versus attentional avoidance), whereas neither attentional strategy influenced the perception of pain in females (Keogh, Hatton, \& Ellery, 2000). Moreover, a recent study showed that noxious heat stimuli produced sex differences in the time course of pain Females reported greater pain than males during initial exposure to noxious stimuli but with repeated exposure experienced less pain and annoyance than males. These results suggest that females experience greater adaptation and habituation to repeated exposure to painful heat stimuli which may allow them to experience less pain over time (Hashmi \& Davis, 2009). The non-painful stimuli used in our study elicited greater SI peak dipole values in females relative to males regardless of condition, suggesting that the prolonged mu suppression observed only in females during the attend to MNS attentional count period cannot be attributed to greater sensitivity to MNS, but instead is reflective of sex differences in processing basic somatosensory information. Sex differences in attentional processing of somatosensory information may help explain why females show greater sensitivity for most pain modalities (Fillingim \& Maixnerm, 1995; Maffiuletti et al., 2008) and are more prone to chronic pain disorders (Fillingim, 2000; Greenspan et al., 2007).

It is unlikely that the sex-specific mu differences reported here are attributable to behavioural differences as both males and females experienced identical task-related sensory input, comparable sensorimotor stimulus thresholds, and similar behavioural scores. Moreover, males and females were comparable on a number of non-physiological factors including age, handedness, and education. However, we cannot discount several limitations of this study including: i) MNS is a non-natural type of somatosensory 
stimulation that is susceptible to sex differences in the perceived level of somatosensory discomfort, ii) the attentional conditions were not adequately equated for stimuli frequency (i.e. predictable MNS events paired with unpredictable video events) or occurrence (i.e. fewer video incidents versus MNS events to count), and ii) we did not control for the gonadal hormones (i.e. females were not matched for menstrual cycle phase) which may have may have affected somatosensory sensitivity and/or brain reactivity (Tu et al., 2009). However, it has been suggested that gonadal hormones do not influence somatosensory sensitivity (Klatzkin, Mechlin, \& Girdler, 2009). Future directions will examine mu rhythms with a paradigm that equally balances the attentional conditions and increases attentional demand requirements (i.e. presentation of random MNS events paired with an equal number of random video events). Moreover, two additional self-rated behavioural measures will be administered to rank the perceived level of tactile discomfort experienced by the paradigm and to report if any additional behavioural or cognitive strategy was used to perform either task.

Our findings further expand the current understanding of mu rhythms and may i) suggest that the $10-12 \mathrm{~Hz}$ mu rhythm is sensitive to the top-down effects of self-directed attention, and ii) be able to unveil neurophysiological sex differences in how males and females process non-painful somatosensory information. Females, but not males, showed intensive and extensive suppression of the mu response, indicating greater activation of the primary somatosensory cortex. These finding suggests that females may process somatosensory information more vigilantly than males, which could help explain the well-document sex bias reported in pain processing. However, these attention-related sex 
differences in mu reactivity will need to be replicated under better experimental control before mu's role in the top down control of attention can be confirmed. In general, these results are informative for interpreting previous attention-related studies that have collapsed across sex, as well as stress the importance delineating between the sexes for future attentional paradigms. Lastly, sex differences in top-down control of attention suggest critical ramifications for psychiatric and neurological illnesses associated with attentional deficits such as Attention Deficit Hyperactivity Disorder.

\subsection{Materials and Methods}

Participants. We studied 20 right-handed healthy control adults (10 females; mean age 25.5 years old and 10 males; mean age 27.8 years old). Participants were recruited using advertisements posted at a university campus in a large metropolitan city. Not surprisingly, therefore, the sample was well-educated: all had completed at least a postsecondary university degree. Participants were excluded if they wore orthodontic braces, had any non-removable metal, had a previous or current diagnosis of psychosis, or a neurological disorder, or were left-handed.

MEG recordings. Sensory fields were recorded with a 151-channel whole head MEG system (VSM MedTech Ltd., Vancouver, Canada). MEG signals were digitized at a sampling rate of $1250 \mathrm{~Hz}$ and an online bandpass filter of $0.3-300 \mathrm{~Hz}$. Prior to recording, each subject was fitted with 3 fiducial localization coils attached to anatomical skin markers placed at the nasion and bilateral preauricular points. Subjects lay in a supine 
position in a magnetically shielded room while head localization measurements were taken at the beginning and end of each experimental condition to determine head position relative to the MEG sensors. Structural magnetic resonance images (MRI) were collected using a 1.5 Tesla Signal Advantage system (GE Medical Systems, Milwaukee, USA) in a $\mathrm{T}_{1}$-weighted sequence. Prior to each MR scan radiographic markers were matched to the fiducial skin markings so that MEG data could be coregistered with the subject's MRI images (Gatez \& Cheyne, 2003; Gatez \& Cheyne, 2006).

Paradigms. Participants lay supine with their head resting in the MEG helmet in the magnetically shielded room while completing two brief controlled attention manipulation tasks. Each experimental task presented identical visual and somatosensory stimuli (a silent but engaging cartoon video, "Pingu the Penguin”; somatosensory stimuli consisting of non-painful electrical pulses applied to the median nerve), and varied only in instructions as to where participants were to direct their attention and which stimulus events to count. Somatosensory stimuli were non-painful, constant-current square wave electrical pulses of 0.2 ms duration delivered by a S88 Dual output square pulse stimulator and PSIU6 Photoelectric isolation unit (Grass Technologies, Astro-Med, Inc.), applied transcutaneously to the right median nerve at a rate of $0.5 \mathrm{~Hz}$ (ISI: 2 s between onset of each stimulus), just above each participants motor threshold (eliciting a small, passive, thumb twitch). There was no sex difference in the mean voltage intensity required to reach motor threshold. Median nerve stimulation (MNS) was presented in a predictable stimulus pattern with impulses delivered in trains of four stimuli followed by a 4s MNS break between trains interval during which no stimulus was presented prior to the next train. Participants were instructed to direct attention to a particular sensory 
modality and mentally count specific task-relevant stimulus events: a) Attend to MNS: subjects were instructed to fixate on the playing video but to attend to the electrical pulses and count the number of MNS omissions that occurred throughout the task. b) Attend away from MNS: subjects were instructed to fixate and attend to the video counting the number of times a specific video incident occurred (Pingu trumpets his bill) while ignoring MNS. MEG conditions were randomized and counter-balanced between sexes. Each task consisted of two 6-minute trials presenting approximately 135 electrical stimuli and 35 MNS omissions.

Behavioural Measures. Two behavioural measures were incorporated to assess task performance: 1) a self-report of number of events counted (MNS break intervals, video events); and 2) a 10-cm visual analog scale to assess subjects’ perceived degree of attentional demand required by each task.

Self-Report of 'attentional counts'. Following each condition, participants reported the number of events they were instructed to count (Pingu trumpets or MNS break intervals). Attentional counts were used to ensure that each participant's attention remained actively engaged throughout each task, and that they all successfully complied with task instructions prior to commencing the next condition.

Self-Report of Perceived Attentional Demand. Once all MEG tasks were completed, participants were asked to fill out two visual analog scales by ranking each task according to the degree of attentional demand it required (from least-to-greatest). 
This study was approved by institutional Research Ethics Board and conducted in compliance with the Declaration of Helsinki. All participants provided informed written consent.

\section{Data analyses.}

Beamformer spatial analysis. The synthetic aperture magnetometry (SAM) beamformer (Robinson \& Vrba, 1998) was used to measure the spatial distribution of mu rhythm activity in the sensorimotor cortex during MNS. To ensure the reliability and validity of our results, we followed the well-established methodological approach of recently conducted studies in our lab (Dockstader et al., 2009). Changes in source power were computed using the SAM differential power statistic (pseudo t) for which a baseline time interval (-0.05 s to 0 s prior to the onset of the first stimulus in a train) was subtracted from an active time interval (0s to 0.05 s following onset of the first stimulus of a train) and bandpass filtered at $1-200 \mathrm{~Hz}$. This computation identified brain areas of peak activation time-locked to the first MNS event in a train that were later used to create 'virtual sensors' by passing the single trial MEG signals through a spatial filter of 1$30 \mathrm{~Hz}$.

Group Spatial Localization. SI peak activity was localized separately for males and females to ensure that any modulatory effects observed could not be attributed to sex differences in source localization. For both groups, source localization was generated using differential SAM analyses of broad-spectrum activity $(1-200 \mathrm{~Hz})$ for both directed attention conditions. Next, each individual's SI peak location was transposed into 
Talairach coordinates using Statistical Parametric Mapping software (Figure 1 A, B) (SPM-Statistical Parametric Mapping. Wellcome Department of Imaging Neuroscience. Available at: http://www.fil.ion.ucl.ac.uk/spm/software).

Time-Frequency Analyses: To investigate the time-frequency specificity of the SI neural response we used the virtual sensor data derived from SAM, and applied a wavelet-based technique (Tallon-Baudry et al., 1997) that averaged SI source power activity across all trials creating time-frequency response (TFR) plots. Virtual sensor data was passed through a spatial filter of $1-30 \mathrm{~Hz}$ and a baseline period of $0.6 \mathrm{~s}$ was used for TFR plots displaying the initial three stimuli events of a train (i.e. $-9 \mathrm{~s}$ to $-8.4 \mathrm{~s}$ prior to the onset of the first stimulus), and the $4 \mathrm{~s}$ MNS omission interval (i.e. $-1 \mathrm{~s}$ to $-1.6 \mathrm{~s}$ prior to stimuli initiating the subsequent train). TFR plots were averaged separately across males and females to create sex-specific grand-averaged images of summed data displaying SI source activity for the ascribed datasets.

Statistical Analyses of the Mu Response. Using the same statistical approach as that of our most recent study (Dockstader et al., 2009), sensorimotor rhythms were first investigated by measuring mu activity (ERD and/or ERS) values that occurred after the first three stimuli of the train for both conditions. Next, we investigated mu responses following the last stimulus in a train.

Bounding Boxes. For the contralateral hemisphere, the frequency boundaries for mu reactivity were always $10-12 \mathrm{~Hz}$ while the temporal boundaries varied according to each dataset. Three temporal boundaries of $0.4 \mathrm{~s}$ each $(-6.8 \mathrm{~s}$ to $-6.4 \mathrm{~s},-4.8 \mathrm{~s}$ to $-4.4 \mathrm{~s},-2.8 \mathrm{~s}$ to - 
2.4s) were chosen for the dataset displaying neural responses following the first three stimuli in the train (Figure 3). One temporal boundary of -1.0 to $0.3 \mathrm{~s}$ (total duration of 1.4s) was chosen for the dataset focusing on the $4 \mathrm{~s}$ break (Figure 5). These time parameters were chosen based on peak neural responses observed in the grand-averaged female TFR plot for the Attend to MNS task for two reasons: 1) attention is directed towards somatosensory stimuli during this condition thus the SI response should be the strongest, and 2) females displayed the most robust SI modulatory effects in this task, thus we tailored our boundary parameters to capitalize on the sex and condition that elicited the strongest sensorimotor reactivity. Next, these time-frequency boundary box parameters were applied to each individual's TFR and the pixel values within that boundary box were averaged separately for males and females to obtain sex-specific peak values for mu activity for each condition. All sex-specific mean values for sensorimotor reactivity were analyzed using the Statistical Package for the Social Sciences (SPSS) version 16.0. Prior to conducting statistical analyses, the data were checked for outliers. Tabachnik and Fidell's conservative score-changing procedure was used for extreme mu scores (defined as $\geq 3$ standard deviations from the group mean) on any variable. Deviant mu scores were adjusted to be within one unit away from the next ranked score, thereby retaining its rank order while minimizing the skew it created in the sample. This procedure was applied to a total of two scores (2 mu scores for the Attend to MNS task derived from two male subjects). For each dataset, we examined contralateral mu responses to MNS as a function of stimulus condition [2 x 2 repeated measures ANOVAs, with Group (Females, Males) as a between-subjects factor and Condition (Attend Away from MNS, Attend to MNS) as a within-subjects factor]. Effect sizes were 
expressed as partial eta squared (partial $\eta^{2}$ ), which is roughly equivalent to $R^{2}$ in multiple regression. Effect size values measured by $R^{2}$ or similar indices can be interpreted as follows: values in the range of $.01, .09$ and .25 are considered small, medium and large effect sizes, respectively (Cohen, 1969). 


\section{PART III GENERAL DISCUSSION}




\subsection{General Discussion Overview}

My study demonstrated that the $10-12 \mathrm{~Hz}$ mu rhythm is sensitive to the top-down effects of self-directed attention. It also showed that the mu rhythm might provide neurophysiological evidence that males and females process non-painful somatosensory information differently. Thus, in this section, I will address relevant theories pertaining to attention-related sex differences in cortical activity (i.e. sex-specific neurocognitive theories of attention). The remainder of the section will discuss potential theoretical implications of my study as well as its relevance to well-documented clinical research of sex biases in pain processing. To conclude I will address the limitations of the study as well as report the proposed future directions for this research.

\subsection{The sex-specific neurocognitive theory of attention}

Tasks of visuospatial processing have shown males and females manifest different brain activity patterns despite no differences in behavioural performance (Butler et al., 2006; Hugdahl et al., 2006; Weiss et al., 2003). Researchers hypothesize that these differential effects may be a result of sex-specific cognitive strategies used to perform such tasks. For example, it has been suggested that females tend to use a 'serial'/ 'route finding' strategy in which visually salient landmarks are used as a reference guide for tasks of spatial navigation. By contrast, it has been proposed that males tend to use an 'orientation'/ 'gestalt strategy' based on global reference points and/or memorization of the general configuration of the environment (Lawton, 1994; Lawton, 1996). Using fMRI and a 3D spatial navigation task, Gron et al., 2000 investigated whether males and females manifest different neural activation when maneuvering through an unfamiliar simulated 
environment. Results indicated that females showed significantly greater activation than males in the left right prefrontal cortex (PFC) and the right inferior and superior parietal lobules while males showed significantly greater activation in the left hippocampus, right parahippocampal gyrus, and the left posterior cingulate. These neurophysiological sex differences were believed to reflect the use of sex-specific strategies. Thus, females showed greater prefrontal cortex activation because the 'online' landmark tracking approach of their navigational efforts placed greater demand on working memory resources. Males showed greater activation of the hippocampal region, which is involved in episodic memory. Thus, it was suggested that males were more likely to use past memories and/or experiences as a guideline for spatial navigation, which in turn allowed multiple cues to be processed simultaneously. If males can process multiple sensory cues without compromising performance, this could explain why the males in my study showed mu synchronization (indicative of cortical deactivation) after every tactile event in the attend to MNS task. Notably, this rapid deactivation of the SI region did not compromise their performance accuracy scores. This suggests that there may be sex differences in neural modulation which allow males to process multiple sensory cues simultaneously. However, it is acknowledged that sex differences in tasks requiring higher-order complex cognitive functions (i.e. visuospatial processing) cannot be extrapolated directly to basic somatosensory processing, and instead should only be used as a contextual reference.

Studies using mental rotation tasks (MRT) have provided further evidence of robust sex differences in brain activity patterns (Butler et al., 2006; Hugdahl et al., 2006; Jordan et 
al., 2002; Thomsen et al., 2000; Weiss et al., 2003). Typically in these tasks, subjects are presented with pairs of visual 3D images and are asked to compare and decide whether the figures are identical or mirror images via keyboard button press while performance is measured by response time and accuracy. A recent fMRI study by Weiss et al., 2003 using a MRT reported differential sex effects in BOLD activation despite no differences in behavioural performance. Males exhibited significantly greater activation in the inferior parietal lobe whereas females show significantly greater activation in right frontal brain regions. These findings are in support of the hypothesis of sex-specific strategies employed during tasks of mental rotation wherein males use a gestault strategy as reflected by parietal activation, while females use a more analytic serial strategy as reflected by right frontal activation (Cabeza \& Nyberg, 2000). Furthermore, another fMRI study using a more basic visuospatial line orientation task revealed sex differences in children aged 7-15 years. Males showed significantly stronger activation relative to females in the right lingual gyrus and the cerebellum (Clements-Stephens, Rimrodt, \& Cutting, 2009). The right lingual gyrus is associated with encoding/decoding spatial memories whereas the cerebellum is involved in motor planning and sequencing. These results support the theory that males utilize brain regions more relevant for visuospatial processing and to a greater degree than females (Hugdahl et al., 2006; Jordan et al., 2002; Thomsen et al., 2000), thus sex-specific strategies may develop in early childhood years. Again, it is tempting to extrapolate these findings of sex differences in tasks of mental rotation and visuospatial processing to help explain the sex differences in somatosensory processing found in my study. However, this generalization cannot be stated explicitly since tasks of mental rotation or visuospatial processing are highly complex cognitive 
experiments with instructional and processing demands that are very different to the cognitive demands that were required for my study.

\subsection{Theoretical implications}

There are several possible interpretations that may explain my findings which may include, but are not limited to: biological sex differences in the brain, gonadotropic hormones, sex chromosomal genes that directly affect brain development, sex differences in societal and psychosocial roles, as well as sex differences in cognitive-affective factors or attentional strategy (Fillingim, 2000). We will discuss our findings in the context of sex-specific cognitive strategies, yet it is cautioned that these interpretations are purely speculative and do not suggest a causal relation between the sex differences in the processing of basic somatosensory and attentional strategy since our paradigm did not permit us to draw this conclusion.

The supposition that males may rely more on gestalt/ systemizing approaches, has important implications for this research study. Males showed a reduced physiological response to MNS and no modulatory effects in mu activity based on task specific attentional demands. Here, the absence of mu modulation in males might be attributable to differences in both cognitive load capacity and/or sensory sensitivity between sexes. If males employ a systemizing approach to processing somatosensory information, it may allow for an increased cognitive load capacity than that of females. Thus, the ability to better categorize relevant versus irrelevant information may encourage less demand on attentional resources. Decreases in cognitive demand have shown to decrease neural 
activation (Dujardin et al., 1993; Van Winsum et al., 1984), and thus may account for the reduced amount of mu suppression observed in males. It is plausible, that males might require a more attentionally demanding load of sensory information to elicit the mu suppression observed in females. By contrast, females expressed strong and prolonged mu suppression when cognitive load was the most challenging (i.e. attend to MNS task), indicative of robust activation of the sensorimotor region (Pfurtscheller et al., 2001). Females have also shown greater neural sensitivity during both resting state (Barry et al., 2005; Barry et al., 2006) and physiological stimulation (Kakigi, \& Shibasaki, 1992) thus may not require as much of an attentional load for sensorimotor activation.

\subsection{Clinical implications}

Studies investigating sex differences in pain processing have shown that a notable bias exits with females exhibiting greater sensitivity to experimentally induced pain stimuli than males (Ellermeier \& Westphal, 1995, Feine, Bushnell, Miron, \& Duncan, 1991; Lund et al., 2005; Maffiuletti et al., 2008; Tall \& Crisp, 2004), and tend to suffer more from chronic pain conditions (Rollman \& Lautenbacher, 2001; Wiesenfeld -Hallin, 2005). Researchers hypothesize that psychological and/or sociocultural factors may account for these differences since females tend to focus more on threatening situations, are more likely monitor their bodily state, and have a higher incidence of emotional disorders including depression and anxiety (Rollman, Abdel-Shaheed, Gillespie, \& Jones, 2004). However, a recent study by Maffiuletti et al., 2008 found that females showed greater pain sensitivity and lower pain tolerance relative to males during cutaneous electrical stimulation, as well as greater sensory and supramotor excitability (i.e. required 
lower electrical stimulation current amplitudes to reach threshold levels). Based on this finding, it is possible that sex differences in pain processing might be more attributable to differences in neurophysiological, rather than psychosocial factors.

Neuroimaging techniques such as fMRI have allowed researchers to examine the neural substrates that are activated during painful experiences. For instance, it is known that painful stimuli activate a series or 'neuromatrix' of cortical areas including the cingulate, insula, as well as the somatosensory and cerebellar regions (Henderson, Bandler, Gandevia, \& Macefield, 2006; Melzack, 1999; Peyron, Laurent, \& Garcia-Larrea, 2000). Additionally, several studies have demonstrated that the processing of afferent painful stimuli recruits different brain circuitry in males and females (Henderson, Gandevia, \& Macefield, 2008). For instance, Paulson and colleagues reported significant sex differences in various brain areas using cutaneous heat stimuli, however, differences in the perception of pain intensity between males and females may have accounted for these effects (Paulson, Minoshima, Morrow, \& Casey, 1998). However, another heat pain study reported significant sex differences in the prefrontal, somatosensory, parietal, cingulate and insula cortices, despite no differences in pain intensity ratings (Derbyshire, Nichols, Firestone, Townsend, \& Jones, 2002). Furthermore, a recent fMRI study found that painful hypertonic saline injections generated activation of the pain neuromatrix as well as sex differences in the mid-cingulate cortex, dorsal lateral prefrontal cortex, hippocampus, and cerebellar cortex, despite no differences in maximal pain intensity ratings (Henderson et al., 2008). Notably, the brain regions activated in this study were in the medial pain pathway, which is believed to be involved with coding affective and/or 
motivational aspects of pain-related experiences (Craig, 2003; Maihofner, Herzner, \& Otto Handwerker, 2006). Sex differences observed might reflect differences in the emotional processing of painful stimuli, which in turn may help clarify the sex bias in pain processing. Clearly, these results indicate that sex differences in pain processing cannot be limited to sex differences in neurophysiological factors. Instead, a thorough understanding of sex biases in pain processing will require the integration of a wide variety of multidisciplinary areas of research.

Attentional strategy may help explain differential sex effects exhibited during the painrelated experiences. A behavioural study by Keogh et al., 2000 found that the manipulation of attention towards and away from painful stimuli produced sex differences in pain perception wherein males benefited from an attentional focus strategy while neither an attentional focus nor avoidance strategy assisted females. More specifically, males showed a greater tolerance to painful stimuli across both directed attention manipulations but benefited more from an attentional focus strategy (i.e. lower sensory pain rating) versus an attentional avoidance strategy (Keogh, Hatton, \& Ellery, 2000). Thus, self-directed attentional control toward afferent somatosensory stimuli seems to influence the subjective pain experience in males, but not in females, which may help explain why females typically show a heightened sensitivity to painful stimuli.

Recently, research has shown that there are sex differences in the mu rhythm response where females commonly elicit stronger mu suppression during action-observation and pain perception than males (Cheng et al., 2006, Cheng et al., 2008a, Yang et al., 2009). 
Additionally, no differences in performance were found suggesting that variation in attentional performance cannot account for the greater mu suppression observed in females. Researchers hypothesize that sex differences in empathy may explain these observed differences with females demonstrating a greater sensitivity to empathetic situations (Yang et al., 2009). However, greater mu suppression in females during observation of non-painful hand actions has also been reported (Cheng et al., 2006, Cheng et al., 2008a, Yang et al., 2009), implying that stronger mu suppression may not be specific to action-observer empathetic mimicry, alone.

The present experiment demonstrated a similar sex-specific finding in the mu response, and the behavioural data showed no discrepancy between males and females in the perceived level of attentional demand required by each task or in performance accuracy. Consequently, our sex-specific modulation effects in the mu response cannot be attributed to behavioural factors, and instead might be indicative of underlying neural differences in the attentional processing of somatosensory information. We suggest here that females may have showed greater mu suppression when attending to somatosensory events and a greater neurophysiological response to non-painful tactile stimuli overall perhaps because of either a more effortful top-down approach and/or greater sensitivity to processing afferent sensory information. Thus, sex differences in the mu response might be more related to how males and females utilize attentional resources rather than a reflection of sex differences in empathetic mimicry (Yang et al. 2009). Sex differences in the top-down control of attention to non-painful somatosensory events suggest pertinent clinical implications related to pain processing, and may help elucidate why females are 
more prone to chronic and clinical pain conditions (Fillingim \& Maixnerm, 1995; Hurley \& Adams, 2008; Keogh \& Denford, 2009).

\subsection{Limitations}

A notable limitation of this study was the use of a non-natural type of sensory stimulus (i.e. MNS) which may cause sex effects in mu reactivity to be stimulus specific. Future studies investigating attention-related sex effects in somatosensory processing should consider using other forms of non-painful somatosensory stimuli. For example, tactile stimulation in the form of finger brushing modulated both the 10 and $20 \mathrm{~Hz}$ sensorimotor oscillations in a healthy control sample (Gaetz \& Cheyne, 2006). Furthermore, selective attention to dot patterns presented to index fingers using mechanical Braille stimulators attenuated lower bandwidth frequencies in somatosensory areas (see Bauer et al., 2006 for stimulus details). These studies suggest that somatosensory processing can be examined via other forms of tactile stimuli and future attention manipulation paradigms should use one (or both) of these methods to investigate if similar findings of sex differences in somatosensory processing can be replicated.

There are two important caveats in the experimental design of the paradigm that may have influenced the findings and which will need to be addressed in future studies: i) the two attentional conditions used were not equally balanced for stimulus frequency (i.e. predictable MNS events paired with unpredictable video events) or for attentional load (i.e. approximately 12 video events to count versus 35 MNS events) which may have confounded the results of this study, and ii) the video clip used was an ongoing visual 
presentation with no time-locked response to examine, thus observation of oscillatory changes in source power could not be analyzed in the visual cortex. Future studies will need to resolve these methodological issues by balancing the frequency of number of modality specific stimulus events (i.e. the same number of random MNS and video events to count), as well as using a visual stimulus that is time-locked to the point in time when a mental count of the relevant visual event is to be updated.

I also did not control for the potential effects of gonadal hormones. Studies have shown that somatosensory and pain sensitivity is influenced by gonadal hormones, (most notably estrogen) (Amandusson \& Blomquest, 2001). Moreover, using fluoro-deoxyglucose positron emission topography, a recent study examined whether pain experienced in primary dysmenorrheal (PDM), a condition characterized by menstrual cramping pain in the lower abdomen without pelvic abnormality, altered neural substrates in the brain. Results found increased brain activity in prefrontal and oribitalfrontal regions and decreased brain activity in sensorimotor regions of the left hemisphere in PDM individuals which was not found in menstrual matched controls. These results suggest that females who suffer from ongoing menstrual pain in PDM show abnormal cerebral metabolism in several brain regions associated with pain processing (Tu et al., 2009). However, it has also been reported that gonadal hormonal factors do not influence somatosensory sensitivity (Klatzkin et al., 2009). While there is evidence both for and against the impact of sex hormones on somatosensory processing, it is important to acknowledge the influence that sex hormones may play in somatosensory sensitivity. The female participants used in this study were not equally matched for hormonal phases with 
respect to their menstrual cycle, thus we cannot rule out the possibility that neurophysiological effects found in females for this study (i.e. the greater N20 response and greater mu suppression when attending to MNS) were not influenced (to some degree) by a hypersensitivity to somatosensory stimulation.

Contrary to our hypothesis of greater mu suppression in the attend to MNS condition, there was no main effect found for condition, perhaps due to the relatively modest attentional demand requirement of the paradigm. Moreover, it has been shown that increases in attentional demand increase the magnitude and duration of mu ERD (Dujardin et al., 1993) however our behavioural measures did not generate significant correlations between mu activity and the ratings of perceived attentional demand. Lastly, we cannot discount the possibility inter-person variability or sex differences in the perception of somatosensory discomfort and future studies will incorporate self-report pain questionnaire to better address this issue.

\subsection{Future Directions}

Further research is required to determine whether sex differences in mu reactivity become more robust if the degree of attention demand is increased and better controlled (i.e. MNS and visual events presented in a randomized manner and the required number of taskrelevant stimuli events will be equally balanced across conditions). Although it is unknown whether estrogen and/or hormonal phases of the menstrual cycle affect mu reactivity, future studies should better control for these factors to address the influence they may have on the perception of somatosensory stimulation. Lastly, two additional 
self-rated behavioural reports might be usefully incorporated to address: i) the perceived level of tactile discomfort experienced by the paradigm in order to confirm that sex differences in mu reactivity are not due to sex differences in the perception of the type of somatosensory stimuli used, and ii) whether or not participants used cognitive strategies to perform either task (i.e. a 'categorization' versus ‘serial processing' approach akin to those in visuospatial tasks) (Butler et al., 2006; Gron et al., 2000; Jordan et al., 2002), to substantiate our current speculation that males and females may use sex-specific cognitive strategies to selectively process basic somatosensory information Clearly, the methological limitations of the current paradigm paired with the small heterogeneous sample do not permit such conclusions to be drawn.

Additionally, I would like to use this novel somatosensory paradigm in individuals diagnosed with ADHD. ADHD is a chronic, prevalent, and impairing mental health disorder, characterized by impaired ability to self-regulate attention, impulsiveness and movement (Harpin, 2005; Kessler, Lane, Stang, \& Van Brunt, 2009; Pelham, Foster, \& Robb, 2007). Despite the exponential growth in ADHD research over the past few decades, neural mechanisms underlying problems in attention, motor and behavioural control, are unclear. Since sensorimotor processing plays an essential role in the development of motor and behavioural control, Tannock and colleagues recently instigated a new line of research investigating sensorimotor processing in adults with ADHD. 
For example, a recent project in the Tannock lab used MEG with a novel manipulation of a well-established paradigm (i.e. MNS) to characterize oscillatory brain patterns underlying sensorimotor processing. Our results indicated that adults with ADHD showed abnormal modulation of brain oscillations in the two dominant cortical rhythms involved in sensorimotor function (mu, beta), but only in response to temporally unpredictable sensory events - no group differences were observed for predictable events (Dockstader et al, 2008; Dockstader et al., 2009). Moreover, significant sex differences were found wherein females showed a greater mu response compared to males across both condition (random/predictable MNS events) and diagnosis (ADHD, control). The findings are provocative, albeit based on small heterogeneous samples, and explanatory accounts remain elusive, thereby warranting further investigation. One alternate (or additional) hypothesis is that the observed differences in somatosensory processing in ADHD may be attributable to the detrimental effects of impairments in higher-order processes (e.g., attention). Hypotheses based on this study would suggest that: i) healthy female adults will show greater attention-related modulation of mu rhythms in contralateral primary sensorimotor cortex, in response to non-painful somatosensory stimuli, compared to healthy adult males and to both male and female adults with ADHD; and ii) in adults with ADHD, males and females will not differ in their attentional modulation of their neural response to non-painful stimuli female controls would elicit greater mu suppression relative to male controls and ADHD individuals (both males and females) when attending to somatosensory stimuli. 
This line of research is novel: to our knowledge, we are the only group using MEG to investigate somatosensory processing in ADHD. Other groups are relying on behavioural methods that do not permit direct measurement of brain activity in situ (during somatosensory processing). Accordingly, one major objective for our future research goals will be to investigate attentional modulation of somatosensory processing in ADHD. 


\section{References}

Adrian, E. D., \& Matthews, B.H.C. (1935). Berger rhythm: potential changes from the occipital lobes in man. Brain 57, 355-385.

Amandusson, A., \& Blomqvist, A. (2001). Estrogen receptors can regulate pain sensitivity. Possible explanation of certain chronic pain conditions. [Abstract] Lakartidningen, 98(15), 1774-1778.

Anderson, J. R. (2004). In: Cognitive psychology and its implications (6th Ed.). Worth Publishers. pp. 519

Babiloni, C., Brancucci, A., Arendt-Nielsen, L., Babiloni, F., Capotosto, P., Carducci, F., et al. (2004). Attentional processes and cognitive performance during expectancy of painful galvanic stimulations: A high-resolution EEG study. Behavioural Brain Research, 152(1), 137-147.

Babiloni, C., Brancucci, A., Capotosto, P.., Arendt-Nielsen, L., Chen, A. C., \& Rossini, P. (2005). Expectency of pain is influenced by motor preparation: a high-resolution EEG study of cortical alpha rhythms. Behavioural Neuroscience, 119, 503-511

Babiloni, C., Brancucci, A., Del Percio, C., Capotosto, P., Arendt-Nielsen, L., Chen, A. C., et al. (2006). Anticipatory electroencephalography alpha rhythm predicts subjective perception of pain intensity. The Journal of Pain: Official Journal of the American Pain Society, 7(10), 709-717.

Babiloni, C., Brancucci, A., Vecchio, F., Arendt-Nielsen, L., Chen, A. C., \& Rossini, P. M. (2006). Anticipation of somatosensory and motor events increases centro-parietal functional coupling: An EEG coherence study. Clinical Neurophysiology: Official Journal of the International Federation of Clinical Neurophysiology, 117(5), 10001008.

Baron-Cohen, S., Knickmeyer, R. C., \& Belmonte, M. K. (2005). Sex differences in the brain: Implications for explaining autism. Science (New York, N.Y.), 310(5749), 819823.

Barry, R. J., Clarke, A. R., McCarthy, R., \& Selikowitz, M. (2006). Age and gender effects in EEG coherence: III. Girls with attention-deficit/hyperactivity disorder. Clinical Neurophysiology: Official Journal of the International Federation of Clinical Neurophysiology, 117(2), 243-251.

Barry, R. J., Clarke, A. R., McCarthy, R., Selikowitz, M., Johnstone, S. J., Hsu, C. I., et al. (2005). Age and gender effects in EEG coherence: II. Boys with attention deficit/hyperactivity disorder. Clinical Neurophysiology: Official Journal of the International Federation of Clinical Neurophysiology, 116(4), 977-984. 
Barry, R.J., \& Beh, H.C. (1972). Desychronization of the alpha rhythm of the EEG as a function of intensity of visual stimulation. Psychological Science, 26, 241-242.

Bastiaansen, M. C., Bocker, K. B., Brunia, C. H., de Munck, J. C., \& Spekreijse, H. (2001). Event-related desynchronization during anticipatory attention for an upcoming stimulus: A comparative EEG/MEG study. Clinical Neurophysiology: Official Journal of the International Federation of Clinical Neurophysiology, 112(2), 393-403.

Bastiaansen, M. C., \& Brunia, C. H. (2001). Anticipatory attention: An event-related desynchronization approach. International Journal of Psychophysiology: Official Journal of the International Organization of Psychophysiology, 43(1), 91-107.

Bauer, M., Oostenveld, R., Peeters, M., \& Fries, P. (2006). Tactile spatial attention enhances gamma-band activity in somatosensory cortex and reduces low-frequency activity in parieto-occipital areas. The Journal of Neuroscience: The Official Journal of the Society for Neuroscience, 26(2), 490-501.

Bender, S., Resch, F., Weisbrod, M., \& Oelkers-Ax, R. (2004). Specific task anticipation versus unspecific orienting reaction during early contingent negative variation. Clinical Neurophysiology: Official Journal of the International Federation of Clinical Neurophysiology, 115(8), 1836-1845.

Borbeley, A. A., Hayaishi, O., Sejnowski, T.J., \& Altman, J. S. (Eds) The Regulation of Sleep (Human Frontier Science Program, Strasbourg, 2000).

Burton, H., Abend, N. S., MacLeod, A. M. K., Sinclair, R. J., Snyder, A., Z., Raichle, M.E. (1999). Tactile attention tasks enhance activation in somatosensory regions of the parietal cortex: A positron emission topography study. Cerebral Cortex, 9, 662674.

Butler, T., Imperato-McGinley, J., Pan, H., Voyer, D., Cordero, J., Zhu, Y. S., et al. (2006). Sex differences in mental rotation: Top-down versus bottom-up processing. NeuroImage, 32(1), 445-456.

Cabeza, R., \& Nyberg, L. (2000). Imaging cognition II: An empirical review of 275 PET and fMRI studies. Journal of Cognitive Neuroscience, 12(1), 1-47.

Capotosto, P., Perrucci, M. G., Brunetti, M., Del Gratta, C., Doppelmayr, M., Grabner, R. H., et al. (2009). Is there "neural efficiency" during the processing of visuo-spatial information in male humans? An EEG study. Behavioural Brain Research, 205(2), 468-474.

Chase, M. H., \& Harper, R. M. (1971). Somatomotor and visceromotor correlates of operantly conditioned 12-14 C-SEC sensorimotor cortical activity.

Electroencephalography and Clinical Neurophysiology, 31(1), 85-92. 
Cheng, Y., Lee, P. L., Yang, C. Y., Lin, C. P., Hung, D., \& Decety, J. (2008a). Gender differences in the mu rhythm of the human mirror-neuron system. PloS One, 3(5), e2113.

Cheng, Y., Yang, C. Y., Lin, C. P., Lee, P. L., \& Decety, J. (2008b). The perception of pain in others suppresses somatosensory oscillations: A magnetoencephalography study. NeuroImage, 40(4), 1833-1840.

Cheng, Y. W., Tzeng, O. J., Decety, J., Imada, T., \& Hsieh, J. C. (2006). Gender differences in the human mirror system: A magnetoencephalography study. Neuroreport, 17(11), 1115-1119.

Clements-Stephens, A. M., Rimrodt, S. L., \& Cutting, L. E. (2009). Developmental sex differences in basic visuospatial processing: Differences in strategy use? Neuroscience Letters, 449(3), 155-160.

Cohen, J., ed. Statistical Power Analysis for the Behavioural Sciences. 1969, Erlsbaum Press.: Hillsdale, NJ.

Coull, J. T. (1998). Neural correlates of attention and arousal: Insights from electrophysiology, functional neuroimaging and psychopharmacology. Progress in Neurobiology, 55(4), 343-361.

Craig, A. D. (2003). Pain mechanisms: Labeled lines versus convergence in central processing. Annual Review of Neuroscience, 26, 1-30.

Crick, F., \& Koch, C. (1990). Some reflections on visual awareness. Cold Spring Harbor Symposia on Quantitative Biology, 55, 953-962.

Crick, F., \& Koch, C. (1990). Some reflections on visual awareness. Cold Spring Harbor Symposia on Quantitative Biology, 55, 953-962.

Debener, S., Herrmann, C. S., Kranczioch, C., Gembris, D., \& Engel, A. K. (2003). Topdown attentional processing enhances auditory evoked gamma band activity. Neuroreport, 14(5), 683-686.

Decety, J., \& Batson, C. D. (2007). Social neuroscience approaches to interpersonal sensitivity. Social Neuroscience, 2(3-4), 151-157.

Decety, J., \& Jackson, P. L. (2004). The functional architecture of human empathy. Behavioral and Cognitive Neuroscience Reviews, 3(2), 71-100.

Decety, J., \& Lamm, C. (2006). Human empathy through the lens of social neuroscience. The Scientific World Journal, 6, 1146-1163. 
Della Penna, S., Torquati, K., Pizzella, V., Babiloni, C., Franciotti, R., Rossini, P. M., et al. (2004). Temporal dynamics of alpha and beta rhythms in human SI and SII after galvanic median nerve stimulation. A MEG study. NeuroImage, 22(4), 1438-1446.

Derbyshire, S. W., Nichols, T. E., Firestone, L., Townsend, D. W., \& Jones, A. K. (2002). Gender differences in patterns of cerebral activation during equal experience of painful laser stimulation. The Journal of Pain: Official Journal of the American Pain Society, 3(5), 401-411.

Destexhe, A., \& Sejnowski, T. J., (2000). Why do we sleep? Brain Research, 886, 208223.

Devos, D., Szurhaj, W., Reyns, N., Labyt, E., Houdayer, E., Bourriez, J. L., et al. (2006). Predominance of the contralateral movement-related activity in the subthalamocortical loop. Clinical Neurophysiology: Official Journal of the International Federation of Clinical Neurophysiology, 117(10), 2315-2327.

Dockstader, C., Gaetz, W., Cheyne, D., \& Tannock, R. (2009). Abnormal neural reactivity to unpredictable sensory events in attention-deficit/hyperactivity disorder. Biological Psychiatry, 66(4), 376-383.

Dockstader, C., Gaetz, W., Cheyne, D., Wang, F., Castellanos, F. X., \& Tannock, R. (2008). MEG event-related desynchronization and synchronization deficits during basic somatosensory processing in individuals with ADHD. Behavioral and Brain Functions: $B B F, 4,8$.

Dujardin, K., Bourriez, J. L., \& Guieu, J. D. (1995). Event-related desynchronization (ERD) patterns during memory processes: Effects of aging and task difficulty. Electroencephalography and Clinical Neurophysiology, 96(2), 169-182.

Dujardin, K., Derambure, P., Defebvre, L., Bourriez, J. L., Jacquesson, J. M., \& Guieu, J. D. (1993). Evaluation of event-related desynchronization (ERD) during a recognition task: Effect of attention. Electroencephalography and Clinical Neurophysiology, 86(5), 353-356.

Ellermeier, W., \& Westphal, W. (1995). Gender differences in pain ratings and pupil reactions to painful pressure stimuli. Pain, 61(3), 435-439.

Elul, R. (1972). The Genesis of the EEG. International Review of Neurobiology, 15, $227-$ 272.

Fadiga, L., \& Craighero, L. (2004). Electrophysiology of action representation. Journal of Clinical Neurophysiology, 21, 157-169. 
Fagan, E. R., Taylor, M. J., \& Logan, W. J. (1987). Somatosensory evoked potentials: Part II. A review of the clinical applications in pediatric neurology. Pediatric Neurology, 3(5), 249-255.

Fan, J., Byrne, J., Worden, M. S., Guise, K. G., McCandliss, B. D., Fossella, J., et al. (2007). The relation of brain oscillations to attentional networks. The Journal of Neuroscience: The Official Journal of the Society for Neuroscience, 27(23), 61976206.

Feine, J. S., Bushnell, M. C., Miron, D., \& Duncan, G. H. (1991). Sex differences in the perception of noxious heat stimuli. Pain, 44(3), 255-262.

Fillingim, R. (2000). Sex, gender, and pain: women and men really are different. Current Review of Pain, 4(1), 24-30.

Fillingim, R.B., Maixnerm, W. (1995). Gender differences in the responses to noxious stimuli. Pain Forum 4, 209-221.

Finnigan, S., Humphreys, M. S., Dennis, S., \& Geffen, G. (2002). ERP 'old/new' effects: Memory strength and decisional factor(s). Neuropsychologia, 40(13), 2288-2304.

Forss, N., Salmelin, R., \& Hari, R. (1994). Comparison of somatosensory evoked fields to airpuff and electric stimuli. Electroencephalography and Clinical Neurophysiology, 92(6), 510-517.

Fries, P., Reynolds, J. H., Rorie, A. E., \& Desimone, R. (2001). Modulation of oscillatory neuronal synchronization by selective visual attention. Science (New York, N.Y.), 291(5508), 1560-1563.

Gaetz, W., \& Cheyne, D. (2003). Localization of human somatosensory cortex using spatially filtered magnetoencephalography. Neuroscience Letters, 340(3), 161-164.

Gaetz, W., \& Cheyne, D. (2006). Localization of sensorimotor cortical rhythms induced by tactile stimulation using spatially filtered MEG. NeuroImage, 30(3), 899-908.

Gallese, V., Fadiga, L., Fogassi, L., \& Rizzolatti, G. (1996). Action recognition in the premotor cortex. Brain, 119, 593-609.

Geary, D.C. (1998). Male, Female: the Evolution of Human Sex Differences. American Psychological Association, Washington, DC.

Greenspan, J., Craft, R., LeResche L., Arendt-Nielsen L., Berkley K., Fillingim R, et al. (2007). Studying sex and gender differences in pain and analgesia: a consensus report. Pain 132, Suppl 1: S26-45. 
Gron, G., Wunderlich, A. P., Spitzer, M., Tomczak, R., \& Riepe, M. W. (2000). Brain activation during human navigation: Gender-different neural networks as substrate of performance. Nature Neuroscience, 3(4), 404-408.

Grunewald-Zuberbier, E., Grunewald, G., Rasche, A., \& Netz, J. (1978). Contingent negative variation and alpha attenuation responses in children with different abilities to concentrate. Electroencephalographic Clinical Neurophysiology, 44, 37-47.

Gunter, T. C., Friederici, A. D., \& Schriefers, H. (2000). Syntactic gender and semantic expectancy: ERPs reveal early autonomy and late interaction. Journal of Cognitive Neuroscience, 12(4), 556-568.

Hagoort, P. (2003). Interplay between syntax and semantics during sentence comprehension: ERP effects of combining syntactic and semantic violations. Journal of Cognitive Neuroscience, 15(6), 883-899.

Hall, J.A. (1984). Nonverbal sex differences. John Hopkins University Press, Baltimore.

Hari, R., Reinikainen, K., Kaukoranta, E., Hamalainen, M., Ilmoniemi, R., Penttinen, A., et al. (1984). Somatosensory evoked cerebral magnetic fields from SI and SII in man. Electroencephalography and Clinical Neurophysiology, 57(3), 254-263.

Hari, R., Salmelin, R., Makela, J. P., Salenius, S., \& Helle, M. (1997). Magnetoencephalographic cortical rhythms. International Journal of Psychophysiology: Official Journal of the International Organization of Psychophysiology, 26(1-3), 51-62.

Hari, R. (2006). Action-perception connection and the cortical mu rhythm. Progressive Brain Research 159, 253-60.

Harpin, V.A., (2005). The effect of ADHD on the life of an individual, their family, and community from preschool to adult life. Archives of Disease in Childhood, 90 Suppl 1, 2-7

Hashmi, J., \& Davis, K. (2009). Women experience greater heat pain adaptation and habituation than man. Pain, 145, 350-357

Henderson, L. A., Bandler, R., Gandevia, S. C., \& Macefield, V. G. (2006). Distinct forebrain activity patterns during deep versus superficial pain. Pain, 120(3), 286296.

Henderson, L. A., Gandevia, S. C., \& Macefield, V. G. (2008). Gender differences in brain activity evoked by muscle and cutaneous pain: A retrospective study of singletrial fMRI data. NeuroImage, 39(4), 1867-1876. 
Herrmann, C. S., \& Knight, R. T. (2001). Mechanisms of human attention: Event-related potentials and oscillations. Neuroscience and Biobehavioral Reviews, 25(6), 465476.

Hsiao, S. S., O'Shaughnessy, D. M., \& Johnson, K. O. (1993). Effects of selective attention on spatial form processing in monkey primary and secondary somatosensory cortex. Journal of Neurophysiology, 70(1), 444-447.

Hugdahl, K., Thomsen, T., \& Ersland, L. (2006). Sex differences in visuo-spatial processing: An fMRI study of mental rotation. Neuropsychologia, 44(9), 1575-1583.

Hurley, R. \& Adams, M. (2008). Sex, gender, and pain: an overview of a complex field. Anaesthesia and Analgesia, 107(1), 309-317.

Huttunen, J., Komssi, S., \& Lauronen, L. (2006). Spatial dynamics of population activities at S1 after median and ulnar nerve stimulation revisited: An MEG study. NeuroImage, 32(3), 1024-1031.

Hyvarinen, J., Poranen, A., Jokinen, Y. (1980). Influence of attentive behaviour on neuronal responses to vibration in primary somatosensory cortex of the monkey. Journal of Neurophysiology, 43, 87-882.

Ioannides, A. A. (2009). Magnetoencephalography (MEG). Methods in Molecular Biology (Clifton, N.J.), 489, 167-188.

Irinki, A., Tanaka, Iwamura, Y. (1996). Attention induced neuronal activity in the monkey somatosensory cortex revealed by pupillometrics. Neuroscience Research, 25, $173-181$

James, W. (1890). In: The Principles of Psychology. New York: Henry Holt, Vol. 1, pp. 403-404

Jensen, O., Kaiser, J., \& Lachaux, J. P. (2007). Human gamma-frequency oscillations are associated with attention and memory. Trends in Neuroscience. 30(7), 317-24

Jensen, O., Gelfand, J., Kounios, J., \& Lisman, J. E. (2002). Oscillations in the alpha band (9-12 Hz) increase with memory load during retention in a short-term memory task. Cerebral Cortex, 12(8), 877-882.

Johansen-Berg, H., Christensen, V., Woolrich, M., \& Matthews, P. M. (2000). Attention to touch modulated activity in both primary and secondary somatosensory cortex. Neuroreport, 11, 1237-1241.

Johansen-Berg, H., \& Lloyd, D. M. (2000). The physiology and psychology of selective attention to touch. Frontiers in Bioscience: A Journal and Virtual Library, 5, D894904. 
Jordan, K., Wustenberg, T., Heinze, H. J., Peters, M., \& Jancke, L. (2002). Women and men exhibit different cortical activation patterns during mental rotation tasks. Neuropsychologia, 40(13), 2397-2408.

Kaiser, J., Hertrich, I., Ackermann, H., \& Lutzenberger, W. (2006). Gamma-band activity over early sensory areas predicts detection of changes in audiovisual speech stimuli. NeuroImage, 30(4), 1376-1382.

Kakigi, R., \& Shibasaki, H. (1992). Effects of age, gender, and stimulus side on the scalp topography of somatosensory evoked potentials following posterior tibial nerve stimulation. Journal of Clinical Neurophysiology: Official Publication of the American Electroencephalographic Society, 9(3), 431-440.

Katayama, J., \& Polich, J. (1998). Stimulus context determines P3a and P3b. Psychophysiology, 35(1), 23-33.

Kessler, R., Lane, M., Stang, P., \& Van Brunt, D. (2009). The prevalence and workplace costs of adult attention deficit hyperactivity disorder in a large manufacturing firm. Psychological Medicine, 39(1), 137-47.

Keogh, E. \& Denford, S. (2009). Sex differences in perceptions of pain coping strategy usage. European Journal of Pain, 13(6), 629-634.

Keogh, E., Hatton, K., \& Ellery, D. (2000). Avoidance versus focused attention and the perception of pain: Differential effects for men and women. Pain, 85(1-2), 225-230.

Kimura, D. (1996). Sex, sexual orientation and sex hormones influence human cognitive function. Current Opinion in Neurobiology, 6, 259-263.

Klatzkin, R., Mechlin, B. \& Girdler, S. (2009). Menstrual cycle phase does not influence gender differences in experimental pain sensitivity. European Journal of Pain, 14(1), 77-82

Klimesch, W. (1997). EEG alpha rhythms and memory processes. International Journal of Psychophysiology, 26, 319-340.

Klimesch, W., Doppelmayr, M., Pachinger, T., \& Ripper, B. (1997). Brain oscillations and human memory: EEG correlates in the upper alpha and theta band. Neuroscience Letters, 238(1-2), 9-12.

Klimesch, W., Doppelmayr, M., Russegger, H., Pachinger, T., \& Schwaiger, J. (1998). Induced alpha band power changes in the human EEG and attention. Neuroscience Letters, 244(2), 73-76.

Klimesch, W., Sauseng, P., \& Hanslmayr, S. (2007). EEG alpha oscillations: The inhibition-timing hypothesis. Brain Research Reviews, 53(1), 63-88. 
Koshino, Y., \& Isaki, K. (1986). Familial occurrence of the mu rhythm. Clinical EEG (Electroencephalography), 17(1), 44-50.

Kuhlman, W. N. (1978). EEG feedback training of epileptic patients: Clinical and electroencephalographic analysis. Electroencephalography and Clinical Neurophysiology, 45(6), 699-710.

Kutas, M., \& Federmeier, K. D. (2000). Electrophysiology reveals semantic memory use in language comprehension. Trends in Cognitive Sciences, 4(12), 463-470.

Ladouceur, C. D., Dahl, R. E., Birmaher, B., Axelson, D. A., \& Ryan, N. D. (2006). Increased error-related negativity (ERN) in childhood anxiety disorders: ERP and source localization. Journal of Child Psychology and Psychiatry, and Allied Disciplines, 47(10), 1073-1082.

Lawton, C.A. (1994). Gender differences in way-finding strategies: relationship to spatial ability and spatial anxiety. Sex Roles, 30, 765-779.

Lawton, C.A. (1996). Strategies for indoor wayfinding: the role of orientation. Journal of Environmental Psychology, 16, 137-145.

Lenz, D., Schadow, J., Thaerig, S., Busch, N. A., \& Herrmann, C. S. (2007). What's that sound? Matches with auditory long-term memory induce gamma activity in human EEG. International Journal of Psychophysiology: Official Journal of the International Organization of Psychophysiology, 64(1), 31-38.

Lin, Y. Y., \& Forss, N. (2002). Functional characterization of human second somatosensory cortex by magnetoencephalography. Behavioural Brain Research, 135(1-2), 141-145.

Lopes da Silva, F. H., \& Pfurtscheller, G. (1999). Basic concepts on EEG synchronization and desychronnization. In: Pfurtscheller, G., Lopes da Silva, F. H. (Eds), Event-Related Desychronization. Handbook of Electroencephalography and Clinical Neurophysiology, 6, Revised Ed Elsevier. Pp, Amsterdam, pp. 3-11.

Lopes da Silva, F. H. (1991). Neural mechanisms underlying brain waves: from neural membranes to networks. Electroencephalography and Clinical Neurophysiology, 79, 81-93.

Lund, I., Lundeberg, T., Kowalski, J., \& Svensson, E. (2005). Gender differences in electrical pain threshold responses to transcutaneous electrical nerve stimulation (TENS). Neuroscience Letters, 375(2), 75-80.

Luu, P., Tucker, D. M., \& Makeig, S. (2004). Frontal midline theta and the error-related negativity: Neurophysiological mechanisms of action regulation. Clinical Neurophysiology: Official Journal of the International Federation of Clinical Neurophysiology, 115(8), 1821-1835. 
Maffiuletti, N. A., Herrero, A. J., Jubeau, M., Impellizzeri, F. M., \& Bizzini, M. (2008). Differences in electrical stimulation thresholds between men and women. Annals of Neurology, 63(4), 507-512.

Maihofner, C., Herzner, B., \& Otto Handwerker, H. (2006). Secondary somatosensory cortex is important for the sensory-discriminative dimension of pain: A functional MRI study. The European Journal of Neuroscience, 23(5), 1377-1383.

Marshall, P. J., Bar-Haim, Y., \& Fox, N. A. (2002). Development of the EEG from 5 months to 4 years of age. Clinical Neurophysiology: Official Journal of the International Federation of Clinical Neurophysiology, 113(8), 1199-1208.

McClure, E. B. (2000). A meta-analytic review of sex differences in facial expression processing and their development in infants, children, and adolescents. Psychological Bulletin, 126(3), 424-453.

Melzack, R. (1999). Pain--an overview. Acta Anaesthesiologica Scandinavica, 43(9), 880-884.

Meyer, E, M., Ferguson, S.S., Zatorre, R. J., Alivisatos, B., Marrett, S., Evans, A. C. Hakim, A. M., (1991). Attention modulates somatosensory cerebral blood flow response to vibrotactile stimulation as measure by positron emission tomography. Annual Neurology, 29, 440-443.

Miller, B. T., \& D'Esposito, M. (2005). Searching for "the top" in top-down control. Neuron, 48(4), 535-538.

Mima, T., Nagamine, T., Nakamura, K., \& Shibasaki, H. (1998). Attention modulates both primary and second somatosensory cortical activities in humans: A magnetoencephalographic study. Journal of Neurophysiology, 80(4), 2215-2221.

Montaron, M. F., Bouyer, J. J., \& Rougeul-Buser, A. (1979). Relationship between attention and mu rhythms in the cat and the monkey (author's transl). [Relations entre l'attention et le rythme mu chez le chat et le singe] Revue d'Electroencephalographie Et De Neurophysiologie Clinique, 9(4), 333-339.

Mulholland, T. (1995). Human EEG, behavioral stillness and biofeedback. International Journal of Psychophysiology: Official Journal of the International Organization of Psychophysiology, 19(3), 263-279.

Muthukumaraswamy, S. D., \& Johnson, B. W. (2004). Changes in rolandic mu rhythm during observation of a precision grip. Psychophysiology, 41(1), 152-156.

Muthukumaraswamy, S. D., Johnson, B. W., \& McNair, N. A. (2004). Mu rhythm modulation during observation of an object-directed grasp. Brain Research.Cognitive Brain Research, 19(2), 195-201. 
Neuhaus, A. H, Opgen-Rhein, C., Urbanek, C., Gross, M., Hahn, E., Ta, T. M., Koehler, S., \& Dettling, M. (2009). Spatiotemporal mapping of sex differences during attentional processing. Human Brain Mapping, 9, 2997-3008.

Neuper, C., \& Pfurtscheller, G. (2001). Event-related dynamics of cortical rhythms: Frequency-specific features and functional correlates. International Journal of Psychophysiology: Official Journal of the International Organization of Psychophysiology, 43(1), 41-58.

Neuper, C., Wortz, M., \& Pfurtscheller, G. (2006). ERD/ERS patterns reflecting sensorimotor activation and deactivation. Progress in Brain Research, 159, 211-222.

Nikolaev, A. R., Ivanitsky, G. A., Ivanitsky, A. M., Posner, M. I., \& Abdullaev, Y. G. (2001). Correlation of brain rhythms between frontal and left temporal (wernicke's) cortical areas during verbal thinking. Neuroscience Letters, 298(2), 107-110.

Nikouline, V. V., Linkenkaer-Hansen, K., Wikstrom, H., Kesaniemi, M., Antonova, E. V., Ilmoniemi, R. J., et al. (2000). Dynamics of mu-rhythm suppression caused by median nerve stimulation: A magnetoencephalographic study in human subjects. Neuroscience Letters, 294(3), 163-166.

Paller, C. J, Campbell, C. M., Edwards, R. R., \& Dobs, A. S. (2009). Sex-based differences in pain perception and treatment. Pain Medicine, 2, 289-99.

Paulson, P. E., Minoshima, S., Morrow, T. J., \& Casey, K. L. (1998). Gender differences in pain perception and patterns of cerebral activation during noxious heat stimulation in humans. Pain, 76(1-2), 223-229.

Pelham, W., Foster, E., \& Robb, J. (2007). The economic impact of attentiondeficit/hyperactivity disorder in children and adolescents. Ambulatory Pediatrics, 7(1) Supp l, 121-31.

Peyron, R., Laurent, B., \& Garcia-Larrea, L. (2000). Functional imaging of brain responses to pain. A review and meta-analysis (2000). Neurophysiologie Clinique = Clinical Neurophysiology, 30(5), 263-288.

Perry, R., Rosen, H., Kramer, J., Beer, J., Levenson, R., \& Miller, B. (2001). Hemispheric dominance for emotions, empathy and social behavior: Evidence from right and left handers with frontotemporal dementia. Neurocase, 7, 145-160.

Pfurtscheller, G. (2001). Functional brain imaging based on ERD/ERS. Vision Research, 41(10-11), 1257-1260.

Pfurtscheller, G., Brunner, C., Schlogl, A., \& Lopes da Silva, F. H. (2006). Mu rhythm (de)synchronization and EEG single-trial classification of different motor imagery tasks. NeuroImage, 31(1), 153-159. 
Pfurtscheller, G., Krausz, G., \& Neuper, C. (2001). Mechanical stimulation of the fingertip can induce bursts of beta oscillations in sensorimotor areas. Journal of Clinical Neurophysiology: Official Publication of the American Electroencephalographic Society, 18(6), 559-564.

Pfurtscheller, G., \& Neuper, C. (1994). Event-related synchronization of mu rhythm in the EEG over the cortical hand area in man. Neuroscience Letters, 174(1), 93-96.

Pfurtscheller, G., Neuper, C., \& Krausz, G. (2000). Functional dissociation of lower and upper frequency mu rhythms in relation to voluntary limb movement. Clinical Neurophysiology: Official Journal of the International Federation of Clinical Neurophysiology, 111(10), 1873-1879.

Pfurtscheller, G., Pregenzer, M., \& Neuper, C. (1994). Visualization of sensorimotor areas involved in preparation for hand movement based on classification of mu and central beta rhythms in single EEG trials in man. Neuroscience Letters, 181(1-2), 4346.

Pfurtscheller, G., Stancak, A., \& Neuper, C. (1996). Event-related synchronization (ERS) in the alpha band--an electrophysiological correlate of cortical idling: A review. International Journal of Psychophysiology: Official Journal of the International Organization of Psychophysiology, 24(1-2), 39-46.

Picton, T. W. (1992). The P300 wave of the human event-related potential. Journal of Clinical Neurophysiology: Official Publication of the American Electroencephalographic Society, 9(4), 456-479.

Pineda, J. A. (2005). The functional significance of mu rhythms: Translating "seeing" and "hearing" into "doing". Brain Research.Brain Research Reviews, 50(1), 57-68.

Preston, S. D., \& de Waal, F. B. (2002). Empathy: Its ultimate and proximate bases. The Behavioral and Brain Sciences, 25(1), 1-71.

Pribram, K. H., \& McGuinness, D. (1975). Arousal, activation, and effort in the control of attention. Psychological Review, 82(2), 116-149.

Reynolds, J. H., \& Chelazzi, L. (2004). Attentional modulation of visual processing. Annual Review of Neuroscience, 27, 611-647.

Rif, J., Hari, R., Hamalainen, M. S., \& Sams, M. (1991). Auditory attention affects two different areas in the human supratemporal cortex. Electroencephalography and Clinical Neurophysiology, 79(6), 464-472.

Rizzolatti, G., Fadiga, L., Fogassi, L., Gallese, V. (1996). Premotor cortex and the recognition of motor actions. Cognitive Brain Research, 3, 131-141. 
Roland, P. E., Meyer, E., Shibasaki, T., Yamamoto, Y. L., Thompson, C. J., (1982). Regional cerebral blood flow changes in cortex and basal ganglia during voluntary movements in normal human volunteers. Journal of Neurophysiology, 48, 467-480.

Robinson, S., \& Vrba, J., Functional neuroimaging by Synthetic Aperture Magnetometry (SAM) in Recent Advances in Biomagnetism, K.M. Yoshimoto, Kuriki, S., Karibe, H., Nakasato N., Editor. 1998, Tohku Univ. Press Sendai, Japan. p. 302305.

Rollman, G. B., Abdel-Shaheed, J., Gillespie, J. M., \& Jones, K. S. (2004). Does past pain influence current pain: Biological and psychosocial models of sex differences. European Journal of Pain (London, England), 8(5), 427-433.

Rollman, G. B., \& Lautenbacher, S. (2001). Sex differences in musculoskeletal pain. The Clinical Journal of Pain, 17(1), 20-24.

Ruby, P., \& Decety, J. (2003). What you believe versus what you thinkthey believe: A neuroimaging study of conceptual perspective-taking. European Journal of Neuroscience, 17, 2475-2480.

Ruby, P., \& Decety, J. (2004). How would you feel versus how doyou think she would feel? A neuroimaging study of perspective takingwith social emotions. Journal of Cognitive Neuroscience, 16, 988-999.

Rueckert, L \& Naybar, N. (2008). Gender differences in empathy: The role of the right hemisphere. Brain and Cognition, 67,162-167.

Ruytjens L., Georgiadis, J. R., Holstege, G., Wit, H. P., Albers, F. W., Willemsen, A. T. (2007). Functional sex differences in human primary auditory cortex. European Journal of Nuclear Medicine Molecular Imaging. 12, 2073-81

Salenius, S., Portin, K., Kajola, M., Salmelin, R., \& Hari, R., (1997a). Cortical control of human motorneuron firing during isometric contraction. Journal of Neurophysiology, 77, 230-237.

Salenius, S., Schnitzler, A., Salmelin, R., Jousmaki, V., \& Hari, R. (1997b). Modulation of human cortical rolandic rhythms during natural sensorimotor tasks. NeuroImage, 5, 221-228.

Salinas, E. \& Sejnowski, T. J. (2001). Correlated neuronal activity and the flow of neural information. Nature Neuroscience Reviews, 2, 539-550.

Salmelin, R., \& Hari, R. (1994). Spatiotemporal characteristics of sensorimotor neuromagnetic rhythms related to thumb movement. Neuroscience, 60(2), 537-550. 
Shishida, K., Hashizume, A., Onoda, K., Okamoto, Y., \& Yamawaki, S. (2006). Enhanced reactivity and delayed recovery of sensorimotor cortex in the novelty seeking personality. Neuropsychobiology, 54(4), 215-225.

Sokolov, A., Pavlova, M., Lutzenberger, W., \& Birbaumer, N. (2004). Reciprocal modulation of neuromagnetic induced gamma activity by attention in the human visual and auditory cortex. NeuroImage, 22(2), 521-529.

SPM-Statistical Parametric Mapping. Wellcome Department of Imaging Neuroscience. Available at http://www.fil.ion.ucl.ac.uk/spm/software. Accessed September 14, 2008

Stancak, A., \& Pfurtscheller, G. (1996). Mu-rhythm changes in brisk and slow self-paced finger movements. Neuroreport, 7(6), 1161-1164.

Stancak, A., Svoboda, J., Rachmanova, R., Vrana, J., Kralik, J., \& Tintera, J. (2003). Desynchronization of cortical rhythms following cutaneous stimulation: Effects of stimulus repetition and intensity, and of the size of corpus callosum. Clinical Neurophysiology: Official Journal of the International Federation of Clinical Neurophysiology, 114(10), 1936-1947.

Steffensen, S. C., Ohran, A. J., Shipp, D. N., Hales, K., Stobbs, S. H., \& Fleming, D. E. (2008). Gender-selective effects of the P300 and N400 components of the visual evoked potential. Vision Research, 48(7), 917-925.

Steriade, M., \& Llinas, R. R. (1988). The functional states of the thalamus and the associated neuronal interplay. Physiological Reviews, 68(3), 649-742.

Stroganova, T. A., Orekhova, E. V., \& Posikera, I. N. (1999). EEG alpha rhythm in infants. Clinical Neurophysiology: Official Journal of the International Federation of Clinical Neurophysiology, 110(6), 997-1012.

Sutherland, M. T., \& Tang, A. C. (2006). Reliable detection of bilateral activation in human primary somatosensory cortex by unilateral median nerve stimulation. NeuroImage, 33(4), 1042-1054.

Szurhaj W., Derambure P., Labyt E., Cassim F., Bourriez J. L., Isnard J., Guieu J. D., \& Mauguière F. (2003). Basic mechanisms of central rhythms reactivity to preparation and execution of a voluntary movement: a stereoelectroencephalographic study. Clinical Neurophysiology, 1,107-19.

Tabachnik, B., Fidell, L. , ed. Using Multivariate Statistics. 5th ed. 2007, Pearson Education (Inc). New York. 
Tall, J. M., \& Crisp, T. (2004). Effects of gender and gonadal hormones on nociceptive responses to intraplantar carrageenan in the rat. Neuroscience Letters, 354(3), 239241.

Tallon-Baudry, C., Bertrand, O., Delpuech, C., \& Pernier, J. (1997). Stimulus specificity of phase-locked and non-phase-locked $40 \mathrm{~Hz}$ visual responses in human. The Journal of Neuroscience: The Official Journal of the Society for Neuroscience, 16(13), 4240-4249.

Tallon-Baudry, C., Bertrand, O., Henaff, M. A., Isnard, J., \& Fischer, C. (2005). Oscillatory gamma-band $(30-70 \mathrm{~Hz})$ activity induced by a visual search task in humans. Journal of Neuroscience, 17, 722-734.

Thomsen, T., Hugdahl, K., Ersland, L., Barndon, R., Lundervold, A., Smievoll, A. I., et al. (2000). Functional magnetic resonance imaging (fMRI) study of sex differences in a mental rotation task. Medical Science Monitor: International Medical Journal of Experimental and Clinical Research, 6(6), 1186-1196.

Tiihonen, J., Hari, R., \& Hamalainen, M. (1989). Early deflections of cerebral magnetic responses to median nerve stimulation. Electroencephalography and Clinical Neurophysiology, 74(4), 290-296.

Tiitinen, H., Sinkkonen, J., Reinikainen, K., Alho, K., Lavikainen, J., \& Naatanen, R. (1993). Selective attention enhances the auditory 40-hz transient response in humans. Nature, 364(6432), 59-60.

Toro, C., Deuschl, G., Thatcher, R., Sato, S., Kufta, C., \& Hallett, M. (1994). Eventrelated desynchronization and movement-related cortical potentials on the ECoG and EEG. Electroencephalography and Clinical Neurophysiology, 93(5), 380-389.

Tu, C. Niddam, D., Chao, H., Liu, R., Hwang R., Yeh, T., \& Hsieh, J. (2009). Abnormal cerebral metabolism during menstrual pain in primary dysmenorrhea. NeuroImage, 47(1), 28-35.

Van Winsum, W., Sergeant, J., \& Geuze, R. (1984). The functional significance of eventrelated desynchronization of alpha rhythm in attentional and activating tasks. Electroencephalography and Clinical Neurophysiology, 58(6), 519-524.

Virji-Babul, N., Moiseev, A., Cheung, T., Weeks, D., Cheyne, D., \& Ribary, U. (2008). Changes in mu rhythm during action observation and execution in adults with Down syndrome: Implications for action representation. Neuroscience Letters, 436(2), 177180.

Wegesin, D. J. (1998). Event-related potentials in homosexual and heterosexual men and women: Sex-dimorphic patterns in verbal asymmetries and mental rotation. Brain and Cognition, 36(1), 73-92. 
Weiss, E., Siedentopf, C. M., Hofer, A., Deisenhammer, E. A., Hoptman, M. J., Kremser, C., et al. (2003). Sex differences in brain activation pattern during a visuospatial cognitive task: A functional magnetic resonance imaging study in healthy volunteers. Neuroscience Letters, 344(3), 169-172.

Wiesenfeld-Hallin, Z. (2005). Sex differences in pain perception. Gender Medicine: Official Journal of the Partnership for Gender-Specific Medicine at Columbia University, 2(3), 137-145.

Woldorff, M. G., Gallen, C. C., Hampson, S. A., Hillyard, S. A., Pantev, C., Sobel, D., et al. (1993). Modulation of early sensory processing in human auditory cortex during auditory selective attention. Proceedings of the National Academy of Sciences of the United States of America, 90(18), 8722-8726.

Womelsdorf, T., \& Fries, P. (2007). The role of neuronal synchronization in selective attention. Current Opinion in Neurobiology, 17(2), 154-160.

Womelsdorf, T., Fries, P., Mitra, P. P., \& Desimone, R. (2006). Gamma-band synchronization in visual cortex predicts speed of change detection. Nature, 439(7077), 733-736.

Womelsdorf, T., Schoffelen, J. M., Oostenveld, R., Singer, W., Desimone, R., Engel, A. K., et al. (2007). Modulation of neuronal interactions through neuronal synchronization. Science (New York, N.Y.), 316(5831), 1609-1612.

Wrobel, A. (2000). Beta activity: a carrier for visual attention. Acta Neurobiology, 60, 247-260.

Yang, C. Y., Decety, J., Lee, S., Chen, C., \& Cheng, Y. (2009). Gender differences in the mu rhythm during empathy for pain: An electroencephalographic study. Brain Research, 1251, 176-184.

Zhuang, P., Toro, C., Grafman, J., Manganotti, P., Leocani, L., \& Hallett, M. (1997). Event-related desynchronization (ERD) in the alpha frequency during development of implicit and explicit learning. Electroencephalography and Clinical Neurophysiology, 102(4), 374-381. 


\section{FIGURES}

\section{Figure 1a: Female versus Male SI Localization: Attend to MNS}

A

Group SAM - Maximum intensity projection (Positive)
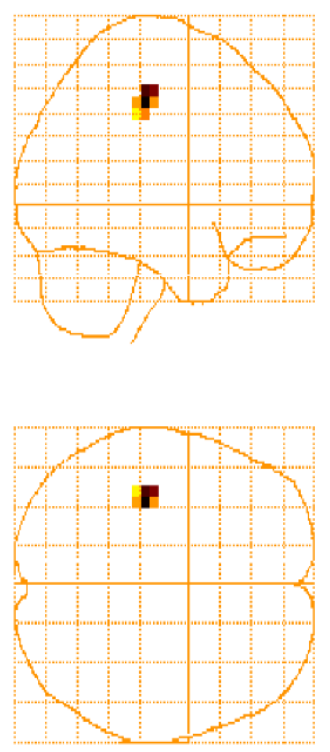

Peak locations $(\mathrm{mm})$ :

Tal coordinates Magnitude $-35-2243$

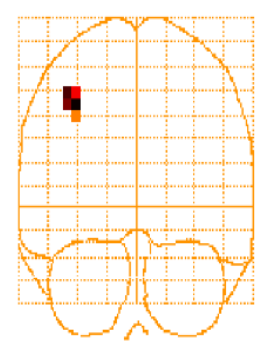

Maximum value $=4.06$

Absolute threshold $=3.25$

Brain Region

Left Postcentral Gyrus (within $0 \mathrm{~mm}$ )
B

Group SAM - Maximum intensity projection (Positive)
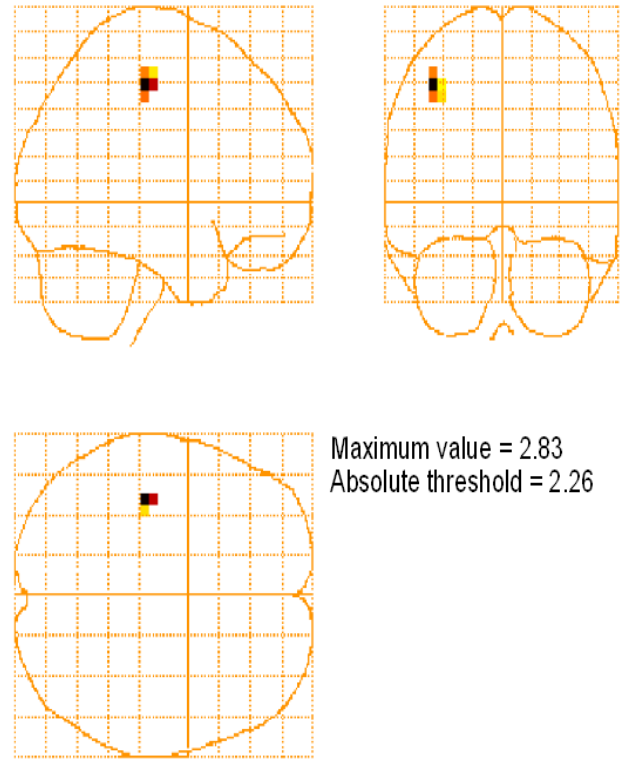

Peak locations ( $\mathrm{mm})$ :

Tal coordinates Magnitude Brain Region $-40-2247 \quad 2.89$
Maximum value $=2.83$

Absolute threshold $=2.26$
Left Postcentral Gyrus (within $0 \mathrm{~mm}$ )

Grand averaged sex-specific mean representations of contralateral SI peaks plotted with Talairach co-ordinate system in SPM.

A. Grand averaged female SI peak activity localized to the left postcentral gyrus

B. Grand averaged male SI peak activity localized to the left postcentral gyrus 


\section{Figure 1b: Female versus Male SI Localization: Attend away from MNS}

A Group SAM - Maximum intensity projection (Positive) $B$
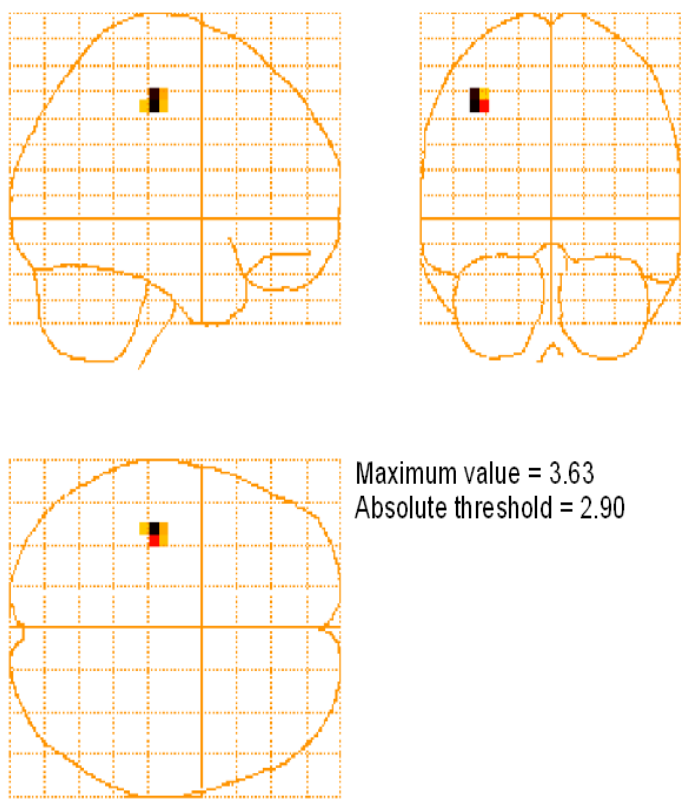

Peak locations $(\mathrm{mm})$ :

Tal coordinates Magnitude $-40-2243$

\section{Maximum value $=3.63$}

Absolute threshold $=2.90$

Absolute threshold $=2.90$

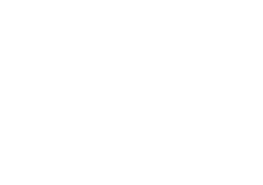

Brain Region
Left Postcentral Gyrus (within $0 \mathrm{~mm}$ )
Group SAM - Maximum intensity projection (Positive)
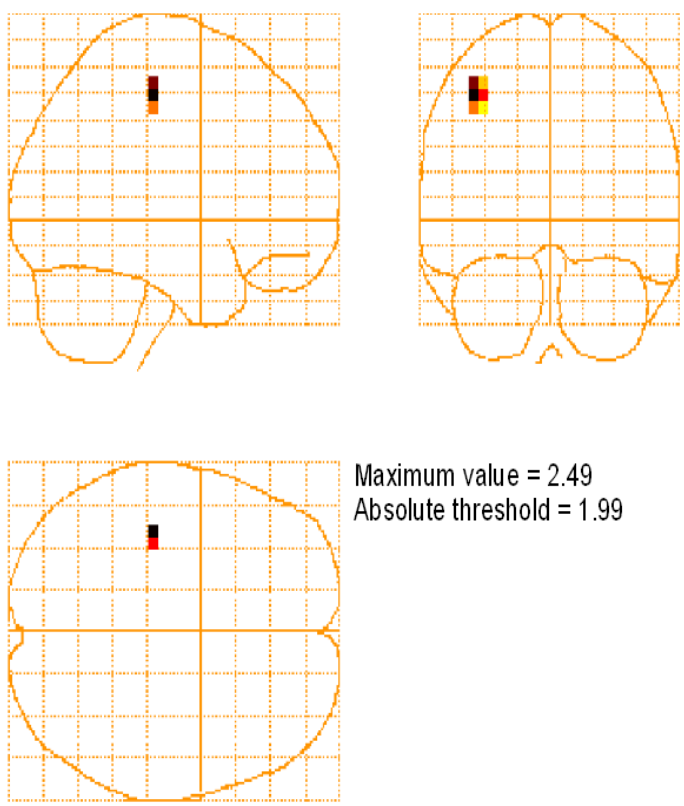

Peak locations $(\mathrm{mm})$ :

Tal coordinates Magnitude Brain Region

$\begin{array}{ccc}-40-2247 & 2.49 & \text { Left Postcentral Gyrus (within } 0 \mathrm{~mm} \text { ) }\end{array}$
Maximum value $=2.49$

Absolute threshold $=1.99$

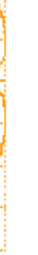

Grand averaged sex-specific mean representations of contralateral SI peaks plotted with Talairach co-ordinate system in SPM.

A. Grand averaged female SI peak activity localized to the left postcentral gyrus

B. Grand averaged male SI peak activity localized to the left postcentral gyrus 


\section{Figure 2: An effective paradigm for observing SI attentional effects}

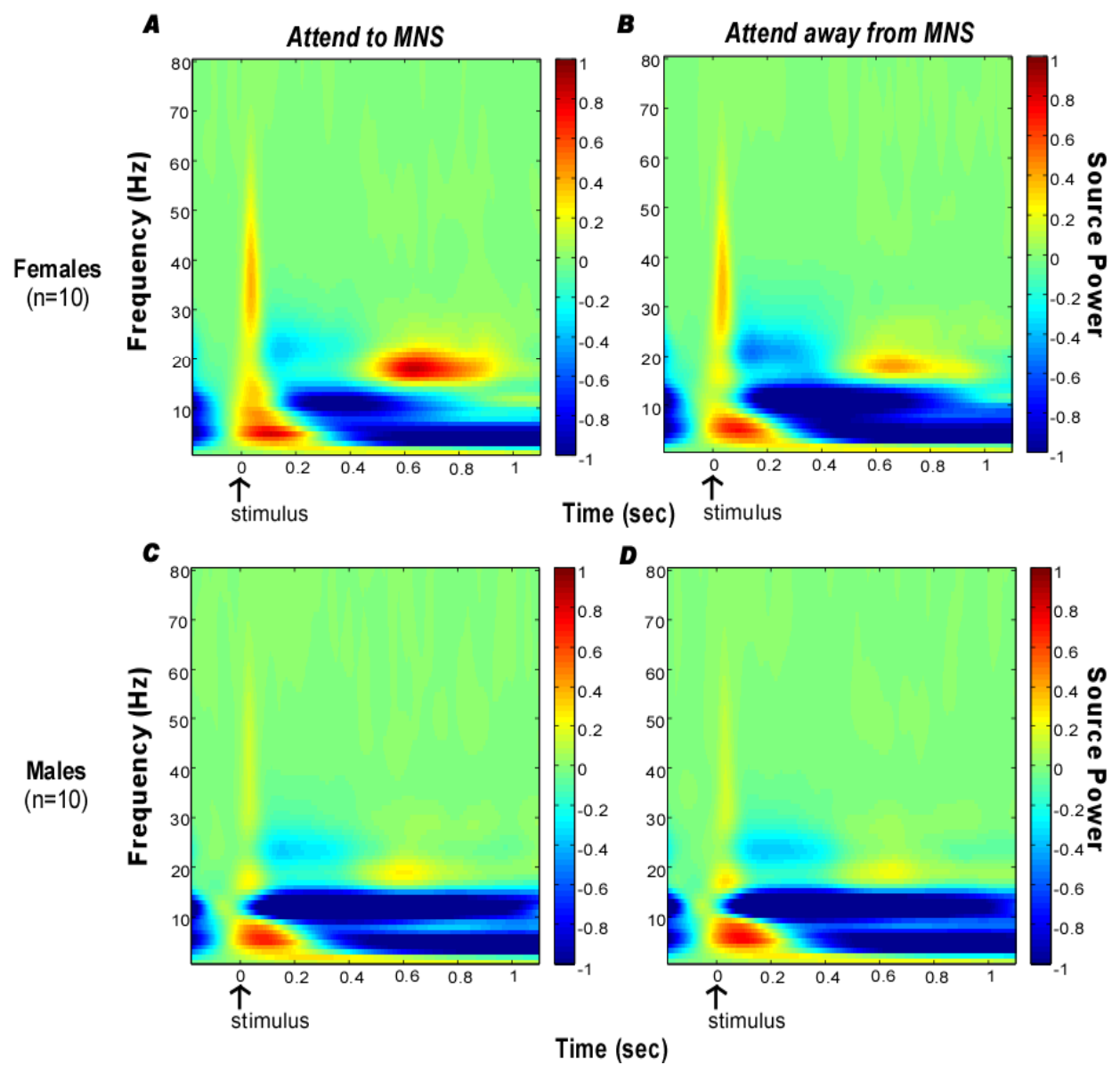

Grand averaged Time Frequency Response (TFR) plots show that both males and females demonstrated the typical pattern of somatosensory activation for each attentional condition:an early, transient, low-frequency $(3-9 \mathrm{~Hz})$ response elicited in the hemisphere contralateral (i.e, left) to the stimulation site followed by an immediate suppression of both the $\sim 10 \mathrm{~Hz}$ and $\sim 20 \mathrm{~Hz}$ mu frequencies and a subsequent burst of post-stimulus $\sim 20 \mathrm{~Hz}$ activity, known as 'beta rebound' (Neuper et al., 2001). A. Grand Mean TFR for female participants during the Attend to MNS task. B. Grand Mean TFR for female participants during the Attend away from MNS task. C. Grand Mean TFR for male participants during the Attend to MNS task. D. Grand Mean TFR for male participants during the Attend away from MNS task. Source Power: A-m2 $=(-n \times 10-17$ to $+n \times 10-17)$. 


\section{Figure 3: Females show prolonged SI activation when attending to somatosensory information}
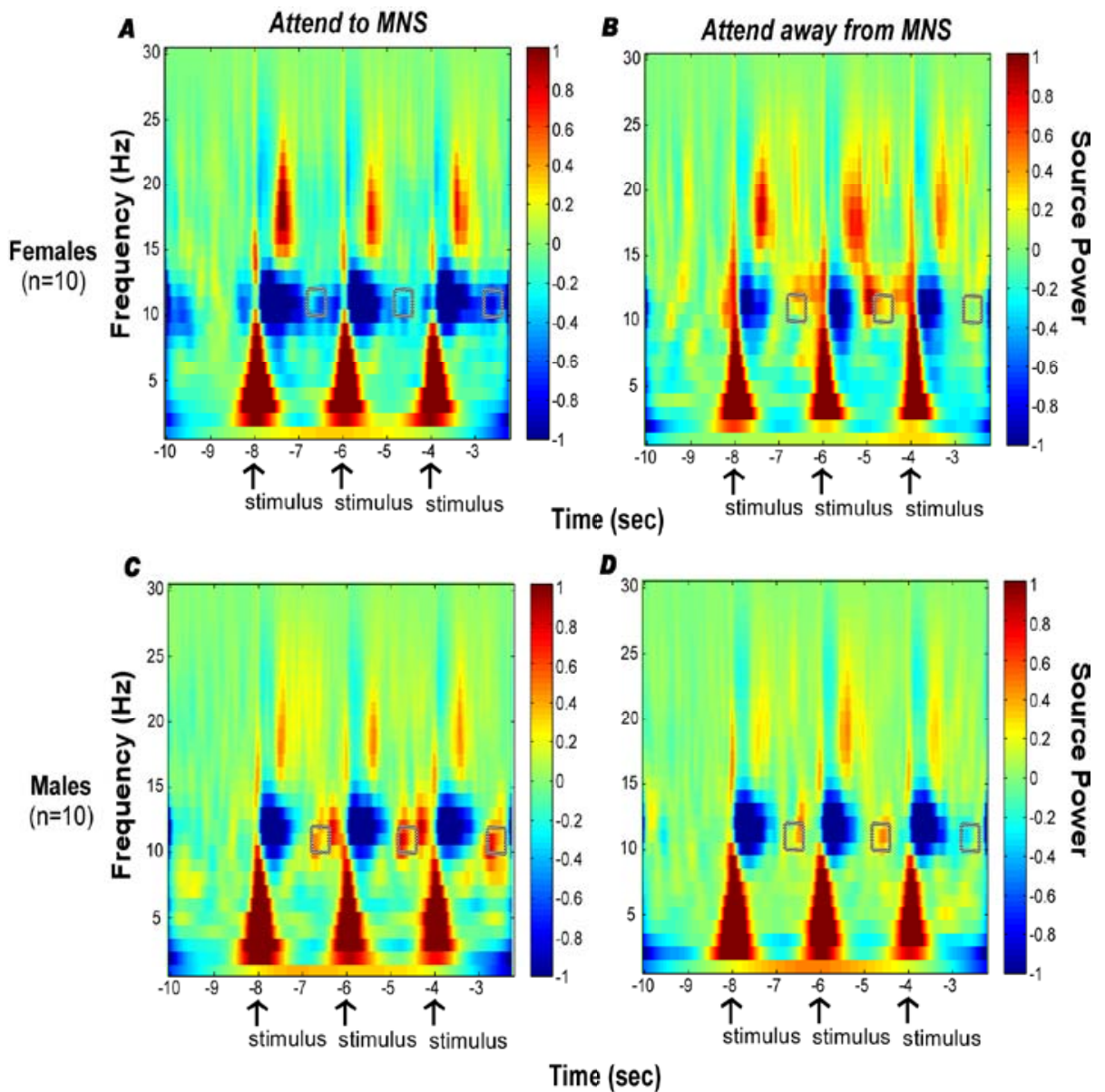

Specific time-frequency boundary boxes of mu activity were defined for the first three stimulus events of a train (Mu box 1: 10$12 \mathrm{~Hz},-6.8 \mathrm{~s}$ to $6.4 \mathrm{~s}$; Mu box 2: $-4.8 \mathrm{~s}$ to $-4.4 \mathrm{~s}$; Mu box $3:-2.8 \mathrm{~s}$ to $-2.4 \mathrm{~s}$ ). Mean values for each boundary box were computed on each individual's TFR data and averaged for each condition. Grand averaged values were used to compare gender-specific effects of attention modulation in SI. A. Grand Mean TFR for female participants during the Attend to MNS task. B. Grand Mean TFR for female participants during the Attend away from MNS task. C. Grand Mean TFR for male participants during the Attend to MNS task. D. Grand Mean TFR for male participants during the Attend away from MNS task. Mu ERD (blue) and ERS (red) responses are delineated with square boxes. Source Power: A-m2 $=(-n \times 10-17$ to $+n \times 10-17)$. 


\section{Figure 4: Grand averaged sex-specific mu reactivity values following the first three stimulus events of a train}

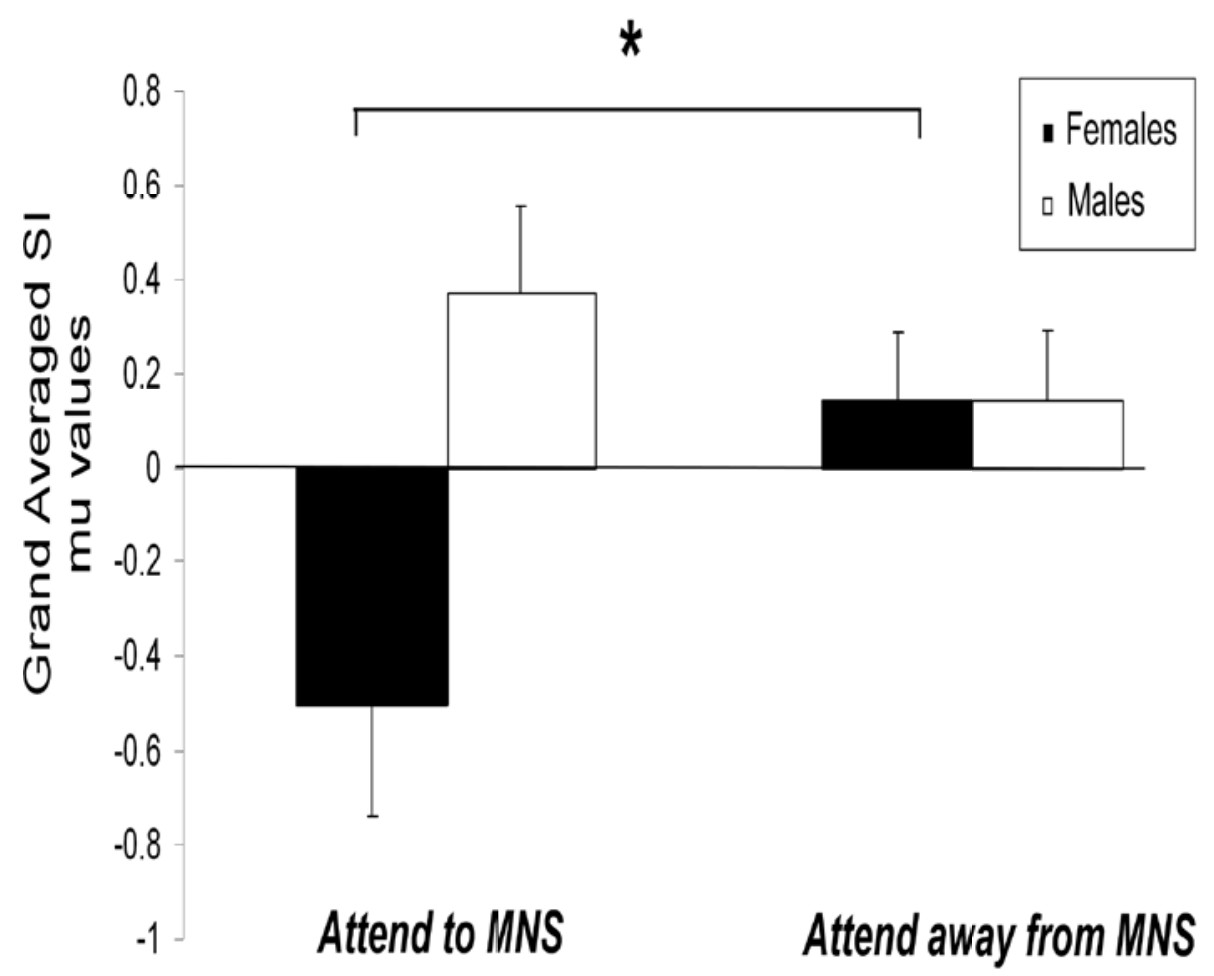

We averaged the mean values of mu activity following the first 3 stimulus events in a train $(10-12 \mathrm{~Hz},-6.8 \mathrm{~s}$ to $-6.4 \mathrm{~s}$, $-4.8 \mathrm{~s}$ to $4.4 \mathrm{~s},-2.8 \mathrm{~s}$ to $-2.4 \mathrm{~s})$. A marginally significant Gender* Condition was found ( $\mathrm{p}=0.057$ ) with females eliciting greater $\mathrm{mu}$ suppression (Mean $=-0.5$ ) versus males (Mean $=0.14)$. 


\section{Figure 5: Sex-specific SI modulation effects of self-directed attention to the 4s MNS break interval}

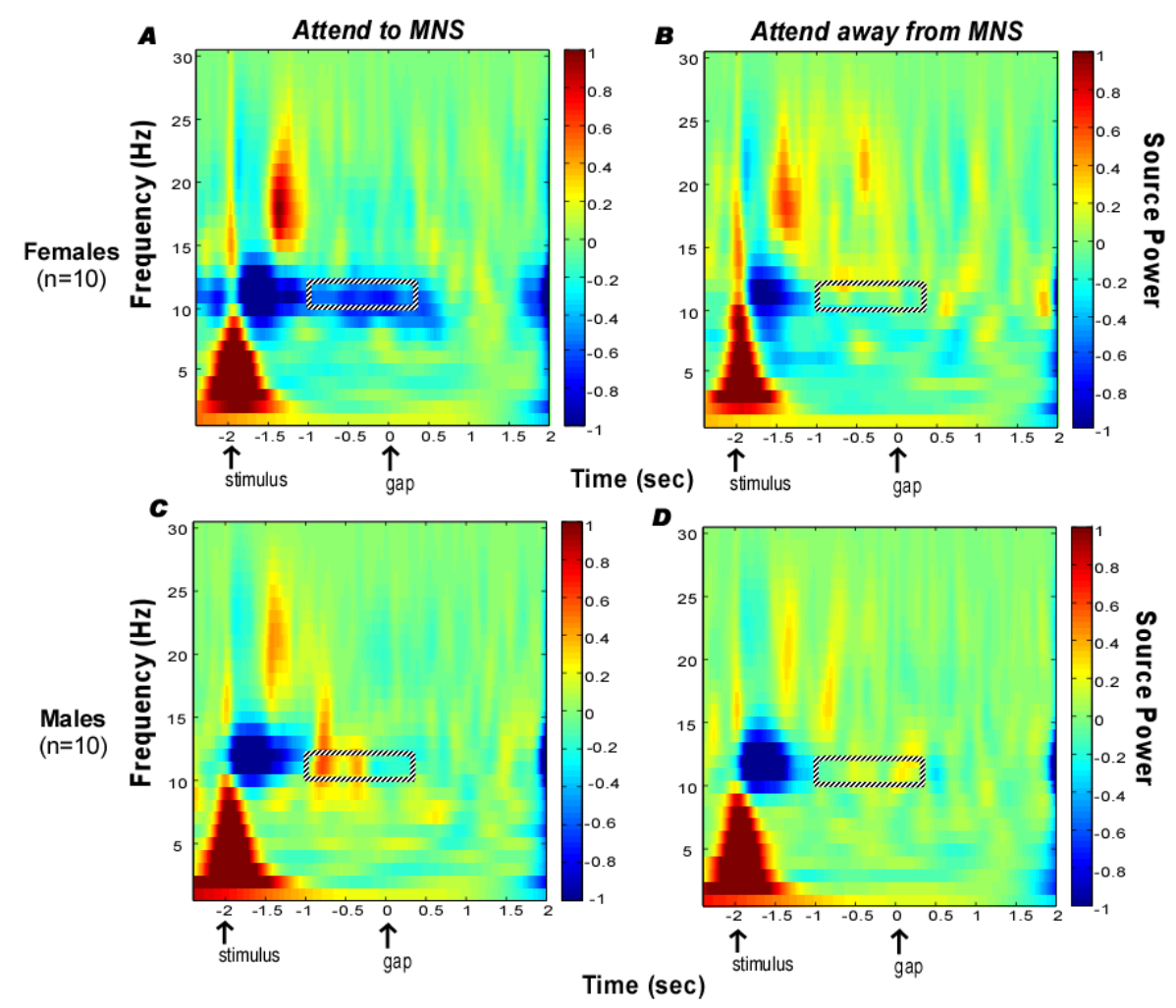

TFR plots reflect both the neural activity in response to the 4 stimulus of a train and the requirement to update the count of the $4 \mathrm{~s}$ MNS gap in the Attend to MNS task but not the Attend away from MNS task. Boundary boxes for mu activity were defined by the Attend to MNS female TFR plot (Mu box: $10-12 \mathrm{~Hz},-1.0$ s to $0.3 \mathrm{~s}$ ). Mean values were computed on each individual's TFR data for both conditions. Grand averaged values were used to compare sex-specific effects of attention modulation in SI. A. Grand Mean TFR for female participants during the Attend to MNS task. B. Grand Mean TFR for female participants during the Attend away from MNS task. C. Grand Mean TFR for male participants during the Attend to MNS task. D. Grand Mean TFR for male participants during the Attend away from MNS task. Mu ERD (blue) and ERS (red) responses are delineated with rectangular boxes. Source Power: A-m2 $=(-n \times 10-17$ to $+n \times 10-17)$. 
Figure 6: Grand-averaged sex-specific mu reactivity values from directedattention to the $4 \mathrm{~s}$ MNS break interval

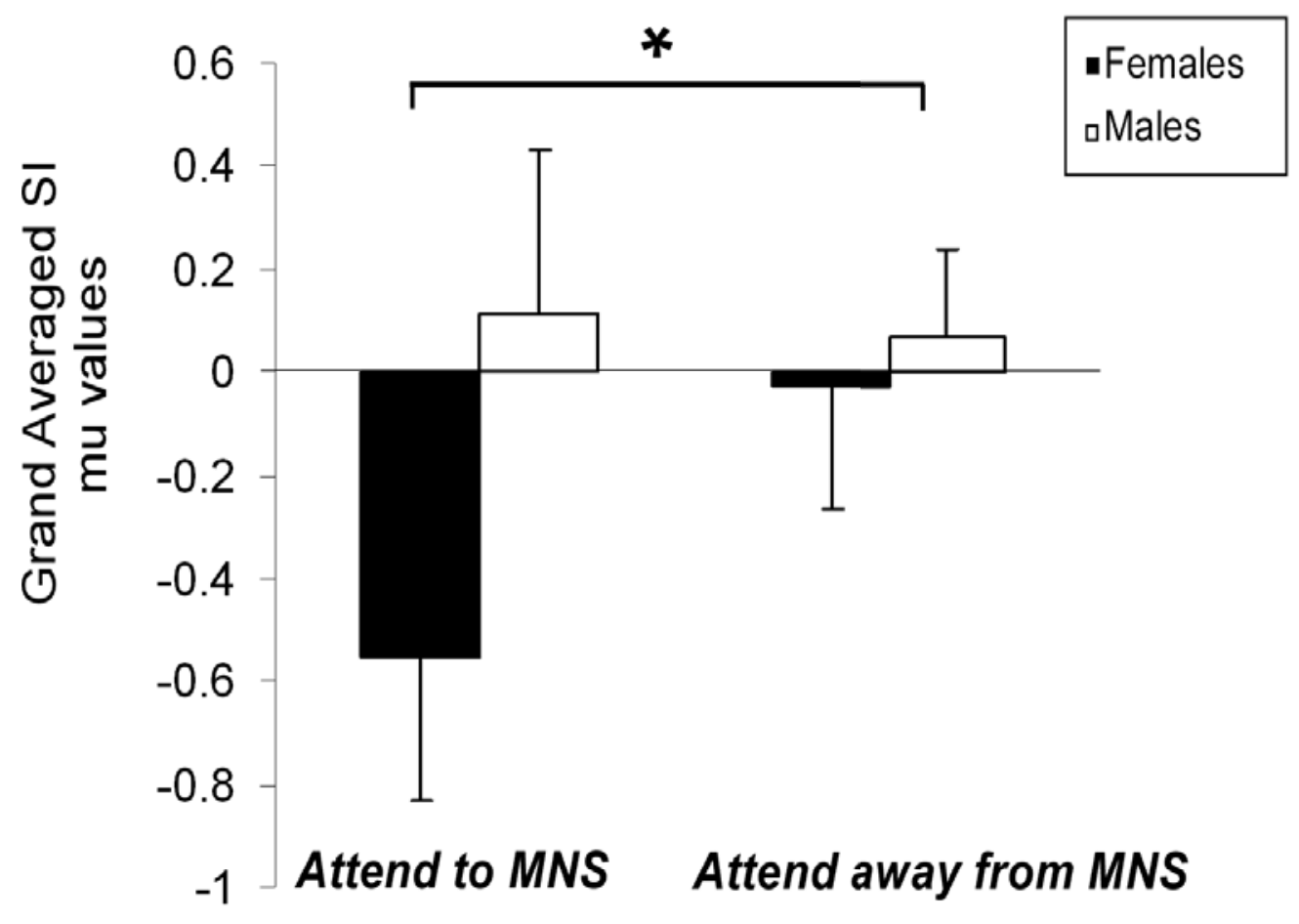

Grand averaged gender specific values for mu activities $(10-12 \mathrm{~Hz},-1.0 \mathrm{~s}$ to $0.3 \mathrm{~s})$ following the fourth stimulus in a train when an attentional count update was required for the Attend to MNS task, but not for the Attend away from MNS task. There was a significant Condition* Gender interaction for late mu activity $(p=0.03)$. Simple t-tests revealed a between group difference with females eliciting stronger mu ERD versus males for the Attend to MNS task, as well as a within-group effect in females between tasks with Attend to MNS eliciting stronger mu ERD than the Attend away from MNS task. 